Pacific

Journal of

Mathematics

RATE OF ATTRACTION FOR A SEMILINEAR WAVE EQUATION WITH VARIABLE COEFFICIENTS AND CRITICAL NONLINEARITIES

FÁGNer Dias ARaruna ANd Flank DaVid Morais BEZERra 


\title{
RATE OF ATTRACTION FOR A SEMILINEAR WAVE EQUATION WITH VARIABLE COEFFICIENTS AND CRITICAL NONLINEARITIES
}

\author{
FÁGner Dias ARARuna And Flank DAVid Morais BEZERra
}

\begin{abstract}
We study the rate of convergence of global attractors and eigenvalues of the family of dissipative semilinear wave equations with variable coefficients $u_{t t}+\Lambda_{\epsilon} u+\Lambda_{\epsilon}^{\delta} u_{t}=f(u)$, where $\Lambda_{\epsilon}$ is the elliptic operator $-\operatorname{div}\left(a_{\epsilon}(x) \nabla\right)$ with $\epsilon \in[0,1]$ and sufficiently smooth coefficients $a_{\epsilon}$, and where $\delta \in\left(\frac{1}{2}, 1\right)$ and the nonlinearity $f$ is a continuously differentiable function satisfying suitable growth conditions. We show that the rate of convergence, as $\epsilon \rightarrow 0^{+}$, of the global attractors of these problems, as well as of their eigenvalues, is proportional to the distance of the coefficients $\left\|a_{\epsilon}-a_{0}\right\|_{L^{\infty}(\Omega)}$.
\end{abstract}

\section{Introduction and main result}

In many theoretical and applied problems, it is important to understand what happens when the solutions varies parameters in the model, and wave equations with variable coefficients arise naturally in mathematical modeling of inhomogeneous media (for example, functionally graded materials or materials with damage induced inhomogeneity) in solid mechanics, electromagnetism, fluid flows through porous media (for example, modeling traveling waves in a inhomogeneous gas; see [Egorov and Shubin 1988; Suggs 2009]), and other areas of physics and engineering.

Nonlinear wave equations arise in quantum mechanics, whereas variants of the form

$$
u_{t t}-\operatorname{div}(a \nabla u)+g\left(u, u_{t}\right)=0
$$

appear in the study of vibrating systems with or without damping, and with or without forcing terms.

The first author was partially supported by INCTMat, CAPES and CNPq (Brasil), grants 307893/20111, 552758/2011-6 and 477124/2012-7. The second author was partially supported by FAPESP grant 11/04166-5, CAPES, and CNPq (Brasil) grant 552758/2011-6.

MSC2010: primary 35J05; secondary 34D45, 41A25.

Keywords: wave equations, variable coefficients, global attractors, rates of convergence. 
In this work, $\epsilon \in[0,1]$, and we consider the following problem associated with a semilinear dissipative wave equation with variable coefficients:

$$
\begin{cases}u_{t t}+\Lambda_{\epsilon} u+\Lambda_{\epsilon}^{\delta} u_{t}=f(u), & t>0, x \in \Omega, \\ u(0, x)=u_{0}(x), u_{t}(0, x)=v_{0}(x), & x \in \Omega, \\ u(t, x)=0, & t \geqslant 0, x \in \partial \Omega,\end{cases}
$$

where $\Omega \subset \mathbb{R}^{N}, N \geqslant 3$, is a bounded domain with boundary $\partial \Omega$ sufficiently regular, $\Lambda_{\epsilon}=-\operatorname{div}\left(a_{\epsilon}(x) \nabla\right)$, and $a_{\epsilon}$ is a real function defined in $\Omega$ satisfying

$$
0<m_{0} \leqslant a_{\epsilon}(x) \leqslant M_{0} \text { for all } x \in \Omega .
$$

Moreover, the functions $a_{\epsilon} \in L^{\infty}(\Omega)$ converge uniformly to $a_{0} \in L^{\infty}(\Omega)$, as $\epsilon \rightarrow 0^{+}$. Also, we will assume that $a_{\epsilon}$ is smooth for all $\epsilon \in[0,1]$. For the system (1-1), let us consider $\delta \in\left(\frac{1}{2}, 1\right)$.

The operators $\Lambda_{\epsilon}^{\delta}:=\left(\Lambda_{\epsilon}^{-\delta}\right)^{-1}$ denote the fractional power operators associated with $\Lambda_{\epsilon}$. Provided that $\Lambda_{\epsilon}$ with domain $D\left(\Lambda_{\epsilon}\right)=H^{2}(\Omega) \cap H_{0}^{1}(\Omega)$ is a sectorial operator with $\operatorname{Re} \sigma\left(\Lambda_{\epsilon}\right)>0$, for any $\alpha \in(0,1)$, it follows by Theorem 1.4.2 in [Henry 1981] that

$$
\Lambda_{\epsilon}^{-\alpha}=\frac{\sin \pi \alpha}{\pi} \int_{0}^{\infty} \lambda^{-\alpha}\left(\lambda I+\Lambda_{\epsilon}\right)^{-1} d \lambda .
$$

On the nonlinearity $f: \mathbb{R} \rightarrow \mathbb{R}$, which is continuously differentiable and bounded, we will give conditions under which the problem (1-7) is globally well posed in $H_{0}^{1}(\Omega) \times L^{2}(\Omega)$ and it has global attractors, in the terminology of [Hale 1988] (following closely Theorem 1.1 and Theorem 1.2 in [Carvalho and Cholewa 2002a]; see also [Carvalho and Cholewa 2002b]): if $\rho \leqslant(N+2) /(N-2)$, there exists a constant $C>0$, independent of $\epsilon$, such that

$$
\left|f\left(s_{1}\right)-f\left(s_{2}\right)\right| \leqslant C\left|s_{1}-s_{2}\right|\left(\left|s_{1}\right|^{\rho-1}+\left|s_{2}\right|^{\rho-1}+1\right)
$$

and

$$
\limsup _{|s| \rightarrow+\infty} \frac{f(s)}{s} \leqslant \mu_{0,1}
$$

with $\mu_{0,1}$ being the first eigenvalue of the $\Lambda_{0}$ in $\Omega$.

In the rest of this paper, we will use $C$ to denote a generic positive constant which may change from line to line (unless otherwise stated).

Since the wave equation does not have dissipative character, we have added a "damping" characterized by the term $\Lambda_{\epsilon}^{\delta} u_{t}^{\epsilon}$ with $\delta \in\left(\frac{1}{2}, 1\right)$. This additional term turns problem (1-1) into a sectorial structure (see [Chen and Triggiani 1989]), however, this gives us an extra difficulty, because it is necessary to perform an analysis of the rate of convergence of fractional derivatives. Although the sectorial 
structure for (1-1) is preserved when the dissipative term presents the optimal power $\delta=\frac{1}{2}$ (see [Chen and Triggiani 1989]), the convergence (with rate) of attractors is an open problem for this case.

Related to this issue, in [Arrieta et al. 2013] the authors proved that the difference $\left\|a_{\epsilon}-a_{0}\right\|_{L^{\infty}(\Omega)}$ can be used to show the rate of convergence of attractors in the context of the heat equation. Nonlinear absorption problems with variable coefficients have been considered by many authors; see [Wu and Li 2011; Suggs 2009] and the references therein. For damped wave equations, several authors have studied existence of global attractors; see [Babin and Vishik 1989; Bruschi et al. 2006; Carvalho and Cholewa 2002a; 2002b; Cholewa and Dlotko 2006; Hale 1988; Webb 1980] and the references therein. We can still cite [Bruschi et al. 2006], where the convergence of attractors was shown, but without explicit rate.

In this work, we will investigate the relationship between the convergence of functions $a_{\epsilon} \in L^{\infty}(\Omega)$, which converge uniformly to $a_{0} \in L^{\infty}(\Omega)$, as $\epsilon \rightarrow 0^{+}$, and the proximity between the perturbed and limit attractors, as well as the convergence of the eigenvalues of the operators associated with the problems in (1-1). The difference $\left\|a_{\epsilon}-a_{0}\right\|_{L^{\infty}(\Omega)}$ will be our measure.

To better explain the results in the paper, we introduce some terminology. Let us consider the Hilbert spaces $Y=Y^{0}:=L^{2}(\Omega), Y^{1 / 2}:=H_{0}^{1}(\Omega), Y^{1}:=D\left(\Lambda_{\epsilon}\right):=$ $\left\{u \in H_{0}^{1}(\Omega): \Lambda_{\epsilon} u \in L^{2}(\Omega)\right\}$ and the Hilbert energy space $X=X^{0}=Y^{1 / 2} \times Y$ equipped with the inner product

$$
\left\langle\left[\begin{array}{l}
\phi \\
\varphi
\end{array}\right],\left[\begin{array}{c}
\bar{\phi} \\
\bar{\varphi}
\end{array}\right]\right\rangle_{X}:=\int_{\Omega} a_{\epsilon}(x) \nabla \phi \nabla \bar{\phi} d x+\int_{\Omega} \varphi \bar{\varphi} d x .
$$

We define the operator $A_{\epsilon}: D\left(A_{\epsilon}\right) \subset X \rightarrow X$ by

$$
A_{\epsilon}\left[\begin{array}{l}
\phi \\
\varphi
\end{array}\right]=\left[\begin{array}{cc}
0 & -I \\
\Lambda_{\epsilon} & \Lambda_{\epsilon}^{\delta}
\end{array}\right]\left[\begin{array}{l}
\phi \\
\varphi
\end{array}\right]:=\left[\begin{array}{c}
-\varphi \\
\Lambda_{\epsilon}^{\delta}\left(\Lambda_{\epsilon}^{1-\delta} \phi+\varphi\right)
\end{array}\right]
$$

and

$$
D\left(A_{\epsilon}\right)=\left\{\left[\begin{array}{l}
\phi \\
\varphi
\end{array}\right] \in Y^{(3 / 2)-\delta} \times Y^{1 / 2} ; \Lambda_{\epsilon}^{1-\delta} \phi+\varphi \in Y^{\delta}\right\}=: X^{1},
$$

with $Y^{\delta}$ denoting the domain of the fractional power operators associated with $\Lambda_{\epsilon}$, that is, $Y^{\delta}:=D\left(\Lambda_{\epsilon}^{\delta}\right)$. Let us consider $Y^{\delta}$ endowed with the graph norm $\|x\|_{Y^{\delta}}=\left\|\Lambda_{\epsilon}^{\delta} x\right\|_{Y}$. Notice that

$$
A_{\epsilon}\left[\begin{array}{l}
\phi \\
\varphi
\end{array}\right]=\left[\begin{array}{c}
-\varphi \\
\Lambda_{\epsilon} \phi+\Lambda_{\epsilon}^{\delta} \varphi
\end{array}\right], \quad\left[\begin{array}{l}
\phi \\
\varphi
\end{array}\right] \in Y^{1} \times Y^{\delta},
$$

where $Y^{1} \times Y^{\delta}$ is a dense subset of $D\left(A_{\epsilon}\right)$.

Notice that the operator $A_{\epsilon}$ with domain $Y^{1} \times Y^{\delta}$ is not a closed operator, unless $\delta=\frac{1}{2}$; see [Chen and Triggiani 1989]. 
Problem (1-1) can be written as

$$
\left\{\begin{array}{l}
w_{t}+A_{\epsilon} w=F(w), \quad t>0 \\
w(0)=w_{0} \in X
\end{array}\right.
$$

with $w=\left[\begin{array}{ll}u & u_{t}\end{array}\right]^{T}$ and the nonlinear map $F: X \rightarrow Y \times Y$ defined by

$$
F\left[\begin{array}{l}
\phi \\
\varphi
\end{array}\right]:=\left[\begin{array}{c}
0 \\
f^{e}(\phi)
\end{array}\right]
$$

where $f^{e}: H_{0}^{1}(\Omega) \rightarrow H^{-1 / 2}(\Omega)$ is the Nemytskiu operator associated with $f$.

We will show that these equations define on the space $X$ a nonlinear semigroup $\left\{T_{\epsilon}(t): t \geqslant 0\right\}$ having global attractors $\mathscr{A}_{\epsilon}, \epsilon \in[0,1]$, and that the rate of convergence of the attractors in the sense of the symmetric Hausdorff distance is given by the order of $\left\|a_{\epsilon}-a_{0}\right\|_{L^{\infty}(\Omega)}^{\theta}$ with $\theta \in\left(0, \frac{1}{2}\right)$.

It is worth noting that the dependence of regular attractors on parameters is a very well-studied and well-understood topic nowadays, especially for the case when the perturbation is also regular (like in our case). Basically, all the necessary technique to handle such perturbations can be found already in the monograph of Babin and Vishik [1989]. However, the problem considered has some interesting peculiarities in a sense unusual for the attractor theory, namely, the presence of the fractional powers of the elliptic operator $\Lambda_{\epsilon}$ as well as the necessity to control the dependence of these powers on the parameter $\epsilon$.

The main purpose of this paper is to give a proof of the following result.

Theorem 1.1. Let $\left\{T_{\epsilon}(t): t \geqslant 0\right\}$ be the gradient nonlinear semigroup associated with (1-7) and let $\mathscr{A}_{\epsilon}$ in $X$ be its global attractor, $\epsilon \in[0,1]$. Then there are constants $C>0$ and $\wp \in\left(0, \frac{1}{2}\right)$, independent of $\epsilon$, such that

$$
\operatorname{dist}\left(\mathscr{A}_{\epsilon}, \mathscr{A}_{0}\right)+\operatorname{dist}\left(\mathscr{A}_{0}, \mathscr{A}_{\epsilon}\right) \leqslant C\left\|a_{\epsilon}-a_{0}\right\|_{L^{\infty}(\Omega)}^{\wp},
$$

where

$$
\operatorname{dist}(A, B):=\sup _{x \in A} \inf _{y \in B}\|x-y\|_{X}, \quad A, B \subset X
$$

is the Hausdorff semidistance between $A$ and $B$ in $X$.

We observe that the Hausdorff semidistance between $A$ and $B$, $\operatorname{dist}(A, B)$, examines how the set $A$ is contained in the set $B$. For example, if $\operatorname{dist}(A, B)=0$ then $A$ is contained in the closure of the set $B$.

The rest of this paper is organized as follows. In Section 2 we show that the linear semigroups of contractions associated to the problems (1-7) are analytic and compact, and that their nonlinear semigroups have global attractors $\mathscr{A}_{\epsilon}$ in $X$. In Section 3 we see that the distance between the semigroups are proportional to a power of the distance between coefficients $a_{\epsilon} \in L^{\infty}(\Omega)$. We study the convergence of the operators $A_{\epsilon}^{-1}$ to $A_{0}^{-1}$. We also make a spectral analysis and we prove that the 
convergence of the eigenvalues of the operators associated to (1-7) is proportional to measure $\left\|a_{\epsilon}-a_{0}\right\|_{L^{\infty}(\Omega)}$. In Section 4 we analyze the convergence of equilibria. In Section 5 we study some important properties of the Nemytskiı operators $F$. We also study the convergence of the operators $A_{\epsilon}-F^{\prime}\left(w_{\epsilon}\right)$, as $w_{\epsilon}$ converges to $w_{0}$ in $X$. In Section 6 we analyze the rate of convergence of equilibria. In Section 7 we study the rate of convergence and attraction of local unstable manifolds of an equilibrium. Finally, in Section 8 we prove the main result of this paper.

\section{Functional setting and background results}

Our main goal in this section is to prove the well-posedness of problem (1-7) in $X$ and to ensure that the nonlinear semigroup generated by (1-7) has global attractor with uniform bounds in $X$. Our approach is inspired by a similar idea from [Carvalho and Cholewa 2002a].

Under the assumption above, it is well known that the operator $\Lambda_{\epsilon}$ is a positive, self-adjoint operator with domain $D\left(\Lambda_{\epsilon}\right)=Y^{1}$. Let us denote by $\left\{e^{-\Lambda_{\epsilon} t}: t \geqslant 0\right\}$ the analytic linear semigroup generated by $-\Lambda_{\epsilon}$ on $Y$, for all $\epsilon \in[0,1]$.

According to [Henry 1981], we still have

$$
\left\|\left(\lambda I+\Lambda_{\epsilon}\right)^{-1}\right\|_{\mathscr{L}(Y)} \leqslant C \max \left\{1,|\lambda|^{-1}\right\}
$$

for some $C>0$ independent of $\epsilon$.

Since $\Lambda_{\epsilon}$ is a sectorial operator with $\left\|e^{-\Lambda_{\epsilon} t}\right\|_{\mathscr{L}(Y)} \leqslant C, C$ independent of $\epsilon$, as a consequence of the moment inequality (see Theorem 1.4.4 in [Henry 1981]), there exists a constant $C>0$ such that

$$
\left\|\Lambda_{\epsilon}^{\alpha} x\right\|_{Y} \leqslant C\left\|\Lambda_{\epsilon} x\right\|_{Y}^{\alpha}\|x\|_{Y}^{1-\alpha}, \quad x \in Y^{1},
$$

with $0 \leqslant \alpha \leqslant 1$. The constant $C$ can be chosen uniform with respect to $\epsilon$ and $\alpha$.

In this way, since all operators are selfadjoint, we have that $\sigma\left(\Lambda_{\epsilon}\right) \subset(-\infty, \alpha]$ for some $\alpha<0$ and, in particular, the set $\Sigma_{\phi}=\{\lambda \in \mathbb{C}:|\arg \lambda| \leqslant \phi\}, \phi \in(\pi / 2, \pi)$, is contained in the resolvent sets of $\Lambda_{\epsilon}$, for all $\epsilon \in[0,1]$. Consequently,

$$
\left\|\Lambda_{\epsilon}\left(\lambda I+\Lambda_{\epsilon}\right)^{-1}\right\|_{\mathscr{L}\left(Y^{1 / 2}\right)} \leqslant C, \quad \lambda \in \Sigma_{\phi},
$$

for some $C \geqslant 1$ independent of $\epsilon$.

We will show that (1-7) is defined on the phase space $X$, an analytic semigroup.

Proposition 2.1. Let $\epsilon \in[0,1]$. The following conditions hold:

(i) The operator $A_{\epsilon}$ is closed.

(ii) $A_{\epsilon}$ is a maximal accretive operator, or equivalently, $-A_{\epsilon}$ is maximal dissipative.

(iii) $0 \in \rho\left(A_{\epsilon}\right)$ and $A_{\epsilon}$ has compact resolvent for each $\epsilon \in[0,1]$. 
(iv) The semigroup linearly generated by $-A_{\epsilon}$ on $X,\left\{e^{-A_{\epsilon} t}: t \geqslant 0\right\}$, is a $C^{0}$ semigroup of contractions on $X$.

(v) $A_{\epsilon}$ is a sectorial operator in $X$ with $\operatorname{Re} \sigma\left(A_{\epsilon}\right)>0$. The semigroup of contractions $\left\{e^{-A_{\epsilon} t}: t \geqslant 0\right\}$ is analytic and compact.

Proof. Note that (i) is immediate from the closedness of $\Lambda_{\epsilon}$ and $\Lambda_{\epsilon}^{\delta}$. For (ii) notice that, given $\left[\begin{array}{ll}\phi & \varphi\end{array}\right]^{T} \in X^{1}$, we have

$$
\begin{aligned}
\left\langle A_{\epsilon}\left[\begin{array}{l}
\phi \\
\varphi
\end{array}\right],\left[\begin{array}{l}
\phi \\
\varphi
\end{array}\right]\right\rangle_{X} \\
=-\langle\varphi, \phi\rangle_{Y^{1 / 2}}+\left\langle\Lambda_{\epsilon} \phi+\Lambda_{\epsilon}^{\delta} \varphi, \varphi\right\rangle_{Y} \\
=-\left\langle\Lambda_{\epsilon}^{1 / 2} \varphi, \Lambda_{\epsilon}^{1 / 2} \phi\right\rangle_{Y}+\left\langle\Lambda_{\epsilon}^{\delta}\left(\Lambda_{\epsilon}^{1-\delta} \phi+\varphi\right), \varphi\right\rangle_{Y} \\
=-\left\langle\Lambda_{\epsilon}^{1 / 2} \varphi, \Lambda_{\epsilon}^{1 / 2} \phi\right\rangle_{Y}+\left\langle\Lambda_{\epsilon}^{1 / 2} \varphi, \Lambda_{\epsilon}^{1 / 2} \phi\right\rangle_{Y}+\left\langle\Lambda_{\epsilon}^{\delta / 2} \varphi, \Lambda_{\epsilon}^{\delta / 2} \varphi\right\rangle_{Y},
\end{aligned}
$$

and hence

$$
\operatorname{Re}\left(A_{\epsilon}\left[\begin{array}{l}
\phi \\
\varphi
\end{array}\right],\left[\begin{array}{l}
\phi \\
\varphi
\end{array}\right]\right\rangle_{X}=\left\langle\Lambda_{\epsilon}^{\delta / 2} \varphi, \Lambda_{\epsilon}^{\delta / 2} \varphi\right\rangle_{Y} \geqslant 0, \quad\left[\begin{array}{l}
\phi \\
\varphi
\end{array}\right] \in X^{1},
$$

which proves accretivity of $A_{\epsilon}$.

Furthermore, for each $\left[\begin{array}{ll}\bar{\phi} & \bar{\varphi}\end{array}\right]^{T} \in X$, the linear equation

$$
\left(I+A_{\epsilon}\right)\left[\begin{array}{l}
\phi \\
\varphi
\end{array}\right]=\left[\begin{array}{l}
\bar{\phi} \\
\bar{\varphi}
\end{array}\right]
$$

is equivalent to the system

$$
\left\{\begin{array}{l}
\phi-\varphi=\bar{\phi} \\
\Lambda_{\epsilon} \phi+\varphi+\Lambda_{\epsilon}^{\delta} \varphi=\bar{\varphi}
\end{array}\right.
$$

or to the equation

$$
\Lambda_{\epsilon} \phi+\Lambda_{\epsilon}^{\delta} \phi+\phi=\bar{\varphi}+\bar{\phi}+\Lambda_{\epsilon}^{\delta} \bar{\phi} .
$$

By elliptic theory, it follows that there exists a unique function $\phi \in Y^{1 / 2}$ with $\Lambda_{\epsilon} \phi \in Y$ satisfying (2-6) and, therefore, for each $\epsilon \in[0,1]$, there exists a unique $\left[\begin{array}{ll}\phi & \varphi\end{array}\right]^{T} \in X^{1}$ solving (2-5).

Concerning $0 \in \rho\left(A_{\epsilon}\right)$, we recall that there exists a bounded inverse operator $A_{\epsilon}^{-1}: X \rightarrow X$ given by

$$
A_{\epsilon}^{-1}=\left[\begin{array}{cc}
\Lambda_{\epsilon}^{-(1-\delta)} & \Lambda_{\epsilon}^{-1} \\
-I & 0
\end{array}\right], \quad \epsilon \in[0,1],
$$

where $\Lambda_{\epsilon}^{-\alpha}$ are bounded inverse operators of $\Lambda_{\epsilon}^{\alpha}$. Thus, the resolvent operator $A_{\epsilon}^{-1}$ is compact, because it takes bounded subsets of $X$ into bounded subsets of $X^{1}$, which is compactly embedded in $X$. This shows (iii). 
The property (iv) that $-A_{\epsilon}, \epsilon \in[0,1]$, generates a $C^{0}$ semigroup of contractions on $X$ follows from the Lummer-Phillips theorem (see [Pazy 1983]) and the observations concerning powers of maximal accretive operators (see [Kato 1976]).

Part (v) follows as a consequence of Theorem 1.1 in [Chen and Triggiani 1989]. Finally, compactness of $\left\{e^{-A_{\epsilon} t}: t \geqslant 0\right\}, \epsilon \in[0,1]$, is then a consequence of compactness of the resolvent operators of $A_{\epsilon}$, and the proof is complete.

Let us denote by $\Gamma$ the boundary of $\Sigma_{\phi}$. The following statements are valid:

$$
\begin{aligned}
e^{-A_{\epsilon} t} & =\frac{1}{2 \pi \mathrm{i}} \int_{\Gamma} e^{\lambda t}\left(\lambda I+A_{\epsilon}\right)^{-1} d \lambda, \\
\left\|e^{-A_{\epsilon} t}\right\|_{\mathscr{L}\left(X^{1}, X\right)} & \leqslant C t^{-1 / 2} e^{-\omega t}, \quad t>0,
\end{aligned}
$$

for some $C>0$ independent of $\epsilon$.

Also, we have 


\section{Resolvent convergence}

In this section we will show the convergence of the resolvent operators $A_{\epsilon}^{-1}$ to $A_{0}^{-1}$, as $\epsilon \rightarrow 0^{+}$, and we will establish that the rate of this convergence is $\left\|a_{\epsilon}-a_{0}\right\|_{L^{\infty}(\Omega)}^{1 / 2}$.

We recall the convergence of the resolvent operators $\Lambda_{\epsilon}^{-1}$ to $\Lambda_{0}^{-1}$, as $\epsilon \rightarrow 0^{+}$, in terms of the difference $\left\|a_{\epsilon}-a_{0}\right\|_{L^{\infty}(\Omega)}$. This was proved in [Arrieta et al. 2013], however, for the sake of completeness, we will sketch a proof.

Lemma 3.1. For $h \in Y$ and $\epsilon \in[0,1]$, let us consider $u^{\epsilon} \in Y^{1}$ a solution of the problem

$$
\begin{cases}-\operatorname{div}\left(a_{\epsilon}(x) \nabla u\right)=h & \text { in } \Omega, \\ u=0 & \text { on } \partial \Omega .\end{cases}
$$

Then there is a constant $C>0$, independent of $\epsilon$, such that

$$
\left\|u^{\epsilon}\right\|_{Y^{1 / 2}} \leqslant C\|h\|_{Y}
$$

and

$$
\left\|u^{\epsilon}-u\right\|_{Y^{1 / 2}} \leqslant C\|h\|_{Y}\left\|a_{\epsilon}-a_{0}\right\|_{\infty} .
$$

Proof. The estimate (3-2) follows from uniform boundedness of $a_{\epsilon}$ and Poincaré's inequality.

The solution of problem (3-1) can be obtained by a minimization procedure. That is, if we define

$$
\lambda_{\epsilon}:=\min _{u \in Y^{1 / 2}}\left\{\frac{1}{2} \int_{\Omega} a_{\epsilon}|\nabla u|^{2} d x-\int_{\Omega} h u d x\right\},
$$

then $\lambda_{\epsilon}$ is attained at $u^{\epsilon}$. Therefore,

$$
\begin{aligned}
\lambda_{\epsilon} & =\frac{1}{2} \int_{\Omega} a_{\epsilon}\left|\nabla u^{\epsilon}\right|^{2} d x-\int_{\Omega} h u^{\epsilon} d x \\
& =\frac{1}{2} \int_{\Omega} a_{\epsilon}\left|\nabla u^{\epsilon}-\nabla u^{0}+\nabla u^{0}\right|^{2} d x-\int_{\Omega} h\left(u^{\epsilon}-u^{0}+u^{0}\right) d x,
\end{aligned}
$$

and, evaluating this expression, using that $u^{\epsilon}$ solves Lemma 3.1, we easily obtain

$$
\lambda_{\epsilon}=\lambda_{0}-\frac{1}{2} \int_{\Omega} a_{\epsilon}(x)\left|\nabla u^{\epsilon}-\nabla u^{0}\right|^{2} d x+\frac{1}{2} \int_{\Omega}\left(a_{\epsilon}(x)-a_{0}(x)\right)\left|\nabla u^{0}\right|^{2} d x
$$

which implies

$$
\lambda_{\epsilon}-\lambda_{0} \leqslant \frac{1}{2} \int_{\Omega}\left(a_{\epsilon}(x)-a_{0}(x)\right)\left|\nabla u^{0}\right|^{2} d x .
$$


On the other hand

$$
\begin{aligned}
\lambda_{0} & :=\min _{u \in Y^{1 / 2}}\left\{\frac{1}{2} \int_{\Omega} a_{0}(x)|\nabla u|^{2} d x-\int_{\Omega} h u d x\right\} \\
& \leqslant \frac{1}{2} \int_{\Omega} a_{0}(x)\left|\nabla u^{\epsilon}\right|^{2} d x-\int_{\Omega} h u^{\epsilon} d x \\
& =\lambda_{\epsilon}+\frac{1}{2} \int_{\Omega}\left(a_{0}(x)-a_{\epsilon}(x)\right)\left|\nabla u^{\epsilon}\right|^{2} d x .
\end{aligned}
$$

With this, we obtain

$$
\lambda_{\epsilon}-\lambda_{0} \geqslant-\frac{1}{2} \int_{\Omega}\left(a_{\epsilon}(x)-a_{0}(x)\right)\left|\nabla u^{\epsilon}\right|^{2} d x,
$$

which combined with (3-2) and (3-6) gives us

$$
\left|\lambda_{\epsilon}-\lambda_{0}\right| \leqslant\left\|a_{\epsilon}-a_{0}\right\|_{L^{\infty}(\Omega)} \sup _{\epsilon \in[0,1]}\left\|u^{\epsilon}\right\|_{Y^{1 / 2}}^{2} \leqslant C\|h\|_{Y}^{2}\left\|a_{\epsilon}-a_{0}\right\|_{L^{\infty}(\Omega)} .
$$

Finally, the estimate (3-3) is obtained by combining (3-5) and (3-7).

Corollary 3.2. The operators $\Lambda_{\epsilon}^{-1}: Y \rightarrow Y^{1 / 2}$ are uniformly bounded and converge uniformly to $\Lambda_{0}^{-1}: Y \rightarrow Y^{1 / 2}$, as $\epsilon \rightarrow 0^{+}$. Furthermore, there exists a positive constant $C>0$, independent of $\epsilon$, such that

$$
\left\|\Lambda_{\epsilon}^{-1}\right\|_{\mathscr{L}\left(Y, Y^{1 / 2}\right)} \leqslant C
$$

and

$$
\left\|\Lambda_{\epsilon}^{-1}-\Lambda_{0}^{-1}\right\|_{\mathscr{L}\left(Y, Y^{1 / 2}\right)} \leqslant C\left\|a_{\epsilon}-a_{0}\right\|_{L^{\infty}(\Omega)} .
$$

The uniform convergence of the operators $\Lambda_{\epsilon}^{-1}$ (see Corollary 3.2 in [Arrieta et al. 2013]) implies the convergence of their spectrum. As a matter of fact, the following result holds.

Proposition 3.3 [Carvalho and Piskarev 2006; Kato 1976]. The following statements hold:

(i) If $\mu_{0} \in \sigma\left(-\Lambda_{0}\right)$, there exists a sequence $\epsilon_{n} \rightarrow 0^{+}$and $\left\{\mu_{n}\right\}$, with $\mu_{n} \in \sigma\left(-\Lambda_{\epsilon_{n}}\right)$, $n \in \mathbb{N}$, such that $\mu_{n} \rightarrow \mu_{0}$, as $n \rightarrow \infty$;

(ii) If for some sequences $\epsilon_{n} \rightarrow 0^{+}$and $\mu_{n} \rightarrow \mu_{0}$, as $n \rightarrow \infty$, with $\mu_{n} \in \sigma\left(-\Lambda_{\epsilon_{n}}\right)$, $n \in \mathbb{N}$, then $\mu_{0} \in \sigma\left(-\Lambda_{0}\right)$.

Moreover, from Lemma 3.4 in [Arrieta et al. 2013], there exists $C>0$, independent of $\epsilon$, such that

$$
\left\|\left(\lambda I+\Lambda_{\epsilon}\right)^{-1}-\left(\lambda I+\Lambda_{0}\right)^{-1}\right\|_{\mathscr{L}\left(Y, Y^{1 / 2}\right)} \leqslant C\left\|a_{\epsilon}-a_{0}\right\|_{L^{\infty}(\Omega)},
$$

for each $\lambda \in \Sigma_{\phi}$. 
Proposition 3.4. The operators $\Lambda_{\epsilon}^{-(1-\delta)}: Y^{1 / 2} \rightarrow Y^{1 / 2}$ are uniformly bounded and converge uniformly to $\Lambda_{0}^{-(1-\delta)}: Y^{1 / 2} \rightarrow Y^{1 / 2}$, as $\epsilon \rightarrow 0$. Furthermore, there exists a positive constant $C>0$, independent of $\epsilon$, such that

$$
\left\|\Lambda_{\epsilon}^{-(1-\delta)}\right\|_{\mathscr{L}\left(Y^{1 / 2}\right)} \leqslant C
$$

and

$$
\left\|\Lambda_{\epsilon}^{-(1-\delta)}-\Lambda_{0}^{-(1-\delta)}\right\|_{\mathscr{L}\left(Y^{1 / 2}\right)} \leqslant C\left\|a_{\epsilon}-a_{0}\right\|_{L^{\infty}(\Omega)}^{1 / 2} .
$$

Proof. Notice that, using (2-2), we get

$$
\left\|\Lambda_{\epsilon}^{-(1-\delta)} u\right\|_{Y^{1 / 2}}=\left\|\Lambda_{\epsilon}^{\delta-1 / 2} u\right\|_{Y} \leqslant C\left\|\Lambda_{\epsilon}^{-1} u\right\|_{Y^{1 / 2}}^{1-(\delta-1 / 2)}\|u\|_{Y^{1 / 2}}^{\delta-1 / 2},
$$

where $C>0$ is uniform with respect to $\epsilon$ and $\delta$. Thus (3-11) follows by (3-8).

Before we prove (3-12), let us observe that (2-2) and (3-11) imply

$$
\begin{aligned}
& \left\|\left(\Lambda_{\epsilon}^{-(1-\delta)}-\Lambda_{0}^{-(1-\delta)}\right) h\right\|_{Y^{1 / 2}} \\
& \quad=\left\|\Lambda_{\epsilon}^{1 / 2}\left(\Lambda_{\epsilon}^{-(1-\delta)}-\Lambda_{0}^{-(1-\delta)}\right) h\right\|_{Y} \\
& \quad \leqslant C\left\|\Lambda_{\epsilon}\left(\Lambda_{\epsilon}^{-(1-\delta)}-\Lambda_{0}^{-(1-\delta)}\right) h\right\|_{Y}^{1 / 2}\left\|\left(\Lambda_{\epsilon}^{-(1-\delta)}-\Lambda_{0}^{-(1-\delta)}\right) h\right\|_{Y}^{1 / 2} \\
& \quad \leqslant C\left\|\Lambda_{\epsilon}\left(\Lambda_{\epsilon}^{-(1-\delta)}-\Lambda_{0}^{-(1-\delta)}\right) h\right\|_{Y}^{1 / 2}\left(\left\|\Lambda_{\epsilon}^{-(1-\delta)} h\right\|_{Y}^{1 / 2}+\left\|\Lambda_{0}^{-(1-\delta)} h\right\|_{Y}^{1 / 2}\right) \\
& \quad \leqslant C\left\|\Lambda_{\epsilon}\left(\Lambda_{\epsilon}^{-(1-\delta)}-\Lambda_{0}^{-(1-\delta)}\right) h\right\|_{Y}^{1 / 2},
\end{aligned}
$$

for some $C>0$ independent of $\epsilon$, and for any $h \in Y$.

To prove (3-12), it follows by (3-13) that it is sufficient to obtain an estimate for the norm $\left\|\Lambda_{\epsilon}\left(\Lambda_{\epsilon}^{-(1-\delta)}-\Lambda_{0}^{-(1-\delta)}\right)\right\| \mathscr{L}(Y)$. In fact, it follows by (1-3) that

$$
\Lambda_{\epsilon}^{-\alpha}-\Lambda_{0}^{-\alpha}=\frac{\sin (\pi \alpha)}{\pi} \int_{0}^{\infty} \lambda^{-\alpha}\left[\left(\lambda I+\Lambda_{\epsilon}\right)^{-1}-\left(\lambda I+\Lambda_{0}\right)^{-1}\right] d \lambda .
$$

Using (3-14) (with $\alpha=1-\delta$ ), we can deduce

$$
\begin{aligned}
\| \Lambda_{\epsilon}\left(\Lambda_{\epsilon}^{-(1-\delta)}\right. & \left.-\Lambda_{0}^{-(1-\delta)}\right) \| \mathscr{L}(Y) \\
& \leqslant \int_{0}^{\infty}\left\|\lambda^{-(1-\delta)} \Lambda_{\epsilon}\left[\left(\lambda I+\Lambda_{\epsilon}\right)^{-1}-\left(\lambda I+\Lambda_{0}\right)^{-1}\right]\right\|_{\mathscr{L}(Y)} d|\lambda| .
\end{aligned}
$$

Notice that the resolvent identity

$$
\begin{aligned}
\left(\lambda I+\Lambda_{\epsilon}\right)^{-1}-(\lambda I & \left.+\Lambda_{0}\right)^{-1} \\
& =\left(\lambda I+\Lambda_{\epsilon}\right)^{-1}\left[I-\left(\lambda I+\Lambda_{\epsilon}\right)\left(\lambda I+\Lambda_{0}\right)^{-1}\right] \\
& =\left(\lambda I+\Lambda_{\epsilon}\right)^{-1}\left[\left(\lambda I+\Lambda_{0}\right)-\left(\lambda I+\Lambda_{\epsilon}\right)\right]\left(\lambda I+\Lambda_{0}\right)^{-1} \\
& =\left(\lambda I+\Lambda_{\epsilon}\right)^{-1}\left[\Lambda_{0}-\Lambda_{\epsilon}\right]\left(\lambda I+\Lambda_{0}\right)^{-1} \\
& =\Lambda_{\epsilon}\left(\lambda I+\Lambda_{\epsilon}\right)^{-1}\left[\Lambda_{\epsilon}^{-1}-\Lambda_{0}^{-1}\right] \Lambda_{0}\left(\lambda I+\Lambda_{0}\right)^{-1}
\end{aligned}
$$


holds, and, by sectoriality of $\Lambda_{\epsilon}$, we have

$$
\left\|\Lambda_{\epsilon}\left(\lambda I+\Lambda_{\epsilon}\right)^{-1}\right\|_{\mathscr{L}(Y)} \leqslant C, \quad \text { for all } \epsilon \in[0,1],
$$

where $C>0$ is independent of $\epsilon$.

Substituting (3-16) into (3-15), we get

(3-18) $\left\|\Lambda_{\epsilon}\left(\Lambda_{\epsilon}^{-(1-\delta)}-\Lambda_{0}^{-(1-\delta)}\right)\right\|_{\mathscr{L}(Y)}$

$$
\begin{aligned}
& \leqslant \int_{0}^{\infty}\left\|\lambda^{-(1-\delta)} \Lambda_{\epsilon} \Lambda_{\epsilon}\left(\lambda I+\Lambda_{\epsilon}\right)^{-1}\left[\Lambda_{\epsilon}^{-1}-\Lambda_{0}^{-1}\right] \Lambda_{0}\left(\lambda I+\Lambda_{0}\right)^{-1}\right\| \mathscr{L}(Y) d|\lambda| \\
& \leqslant \int_{0}^{\infty}|\lambda|^{-(1-\delta)}\left\|\Lambda_{\epsilon}^{\gamma}\left(\lambda I+\Lambda_{\epsilon}\right)^{-1} \Lambda_{\epsilon}^{1+(1-\gamma)}\left[\Lambda_{\epsilon}^{-1}-\Lambda_{0}^{-1}\right] \Lambda_{0}\left(\lambda I+\Lambda_{0}\right)^{-1}\right\| \mathscr{L}(Y) d|\lambda|,
\end{aligned}
$$

where $\gamma \in(1,2)$ is a constant to be chosen.

Since $Y^{1 / 2}$ is continuously embedded in $Y^{1+(1-\gamma)}$, by estimates (3-9) and (3-17), we can deduce from (3-18) that

$$
\begin{aligned}
& \left\|\Lambda_{\epsilon}\left(\Lambda_{\epsilon}^{-(1-\delta)}-\Lambda_{0}^{-(1-\delta)}\right)\right\| \mathscr{L}(Y) \\
& \quad \leqslant C\left\|a_{\epsilon}-a_{0}\right\|_{L^{\infty}(\Omega)} \int_{0}^{\infty}|\lambda|^{-(1-\delta)}\left\|\Lambda_{\epsilon}^{\gamma-r} \Lambda_{\epsilon}^{r}\left(\lambda I+\Lambda_{\epsilon}\right)^{-1}\right\| \mathscr{L}(Y) d|\lambda|,
\end{aligned}
$$

where $r \in(\gamma-1,1)$ is a constant to be chosen.

From (2-2) and the fact that $Y^{1}$ is continuously embedded in $Y^{r}$, it follows by (3-19) that

(3-20) $\left\|\Lambda_{\epsilon}\left(\Lambda_{\epsilon}^{-(1-\delta)}-\Lambda_{0}^{-(1-\delta)}\right)\right\|_{\mathscr{L}(Y)}$

$\leqslant C\left\|a_{\epsilon}-a_{0}\right\|_{L^{\infty}(\Omega)} \int_{0}^{\infty}|\lambda|^{-(1-\delta)}\left\|\Lambda_{\epsilon} \Lambda_{\epsilon}^{r}\left(\lambda I+\Lambda_{\epsilon}\right)^{-1}\right\|_{\mathscr{L}(Y)}^{\gamma-r}$

$$
\times\left\|\Lambda_{\epsilon}^{r}\left(\lambda I+\Lambda_{\epsilon}\right)^{-1}\right\|_{\mathscr{L}(Y)}^{1-(\gamma-r)} d|\lambda|
$$

$\leqslant C\left\|a_{\epsilon}-a_{0}\right\|_{L^{\infty}(\Omega)} \int_{0}^{\infty}|\lambda|^{-(1-\delta)}\left\|\Lambda_{\epsilon}\left(\lambda I+\Lambda_{\epsilon}\right)^{-1}\right\|_{\mathscr{L}(Y)}^{\gamma-r}\left\|\left(\lambda I+\Lambda_{\epsilon}\right)^{-1}\right\|_{\mathscr{L}(Y)}^{1-(\gamma-r)} d|\lambda|$.

Using (2-1) and (3-17), we get by (3-20) that

$$
\begin{aligned}
& \left\|\Lambda_{\epsilon}\left(\Lambda_{\epsilon}^{-(1-\delta)}-\Lambda_{0}^{-(1-\delta)}\right)\right\|_{\mathscr{L}(Y)} \\
& \quad \leqslant C\left\|a_{\epsilon}-a_{0}\right\|_{L^{\infty}(\Omega)} \int_{0}^{\infty}|\lambda|^{-(1-\delta)}\left\|\left(\lambda I+\Lambda_{\epsilon}\right)^{-1}\right\|_{\mathscr{L}(Y)}^{1-(\gamma-r)} d|\lambda| \\
& \quad \leqslant C\left\|a_{\epsilon}-a_{0}\right\|_{L^{\infty}(\Omega)} \int_{0}^{\infty}|\lambda|^{-(1-\delta)}\left(\max \left\{1,|\lambda|^{-1}\right\}\right)^{1-(\gamma-r)} d|\lambda| \\
& \quad \leqslant C\left\|a_{\epsilon}-a_{0}\right\|_{L^{\infty}(\Omega)}\left(\int_{0}^{1}|\lambda|^{\delta-1} d|\lambda|+\int_{1}^{\infty}|\lambda|^{-2+\delta+\gamma-r} d|\lambda|\right) .
\end{aligned}
$$


Taking $\gamma$ and $r$ sufficiently close to 1 such that $\delta+\gamma-r<1$, we can conclude by (3-21) the existence of a positive constant $C$, independent of $\epsilon$, such that

$$
\left\|\Lambda_{\epsilon}\left(\Lambda_{\epsilon}^{-(1-\delta)}-\Lambda_{0}^{-(1-\delta)}\right)\right\|_{\mathscr{L}(Y)} \leqslant C\left\|a_{\epsilon}-a_{0}\right\|_{L^{\infty}(\Omega)} .
$$

Finally, combining (3-13) and (3-22) we obtain the desired estimate (3-12).

Before we prove analogous result of the last proposition with $A_{\epsilon}$ instead of $\Lambda_{\epsilon}$, we present the following general version of the moment inequality (see [Sobolevskii 1961]):

$$
\left\|A_{\epsilon} w\right\|_{X} \leqslant C\|w\|_{X}^{1-\beta / \alpha}\left\|A_{\epsilon}^{\alpha} w\right\|_{X}^{\beta / \alpha}, \quad w \in D\left(A_{\epsilon}^{\alpha}\right),
$$

where the constant $C>0$ is independent of $\epsilon$.

Proposition 3.5. The operators $A_{\epsilon}^{-1}: X \rightarrow X^{1}$ are uniformly bounded and converge in the uniform topology to $A_{0}^{-1}: X \rightarrow X^{1}$, as $\epsilon \rightarrow 0^{+}$. Furthermore, there exists a positive constant $C>0$, independent of $\epsilon$, such that

$$
\left\|A_{\epsilon}^{-1}\right\|_{\mathscr{L}\left(X, X^{1}\right)} \leqslant C
$$

and

$$
\left\|A_{\epsilon}^{-1}-A_{0}^{-1}\right\|_{\mathscr{L}\left(X, X^{1}\right)} \leqslant C\left\|a_{\epsilon}-a_{0}\right\|_{L^{\infty}(\Omega)}^{1 / 2} .
$$

Proof. For $g, h \in L^{2}(\Omega)$ and $\epsilon \in[0,1]$, let $\left[\phi_{\epsilon} \varphi_{\epsilon}\right]^{T}$ be the solution of the problem

$$
A_{\epsilon}\left[\begin{array}{l}
\phi \\
\varphi
\end{array}\right]=\left[\begin{array}{l}
g \\
h
\end{array}\right], \quad t>0 .
$$

By (1-6), there exists $C>0$, independent $\epsilon$, such that

$$
\left\|\left[\begin{array}{l}
\phi_{\epsilon} \\
\varphi_{\epsilon}
\end{array}\right]-\left[\begin{array}{l}
\phi_{0} \\
\varphi_{0}
\end{array}\right]\right\|_{X^{1}} \leqslant C\left\|\left[\begin{array}{l}
\phi_{\epsilon} \\
\varphi_{\epsilon}
\end{array}\right]-\left[\begin{array}{l}
\phi_{0} \\
\varphi_{0}
\end{array}\right]\right\|_{Y^{1} \times Y^{\delta}}
$$

and by (3-23), we get

$$
\begin{aligned}
\|\left[\begin{array}{c}
\phi_{\epsilon} \\
\varphi_{\epsilon}
\end{array}\right]-\left[\begin{array}{c}
\phi_{0} \\
\varphi_{0}
\end{array}\right] & \|_{Y^{1} \times Y^{\delta}} \\
& =\left\|\Lambda_{\epsilon}^{-(1-\delta)} g-\Lambda_{0}^{-(1-\delta)} g\right\|_{Y^{1}}+\left\|\Lambda_{\epsilon}^{-1} h-\Lambda_{0}^{-1} h\right\|_{Y^{\delta}} \\
& \leqslant C\left\|\Lambda_{\epsilon}^{1 / 2}\left(\Lambda_{\epsilon}^{-(1-\delta)} g-\Lambda_{0}^{-(1-\delta)} g\right)\right\|_{Y}+\left\|\Lambda_{\epsilon}^{-(1-\delta)} h-\Lambda_{0}^{-(1-\delta)} h\right\|_{Y} .
\end{aligned}
$$

Thus, by Corollary 3.2, we conclude that

$$
\begin{aligned}
\left\|\left[\begin{array}{c}
\phi_{\epsilon} \\
\varphi_{\epsilon}
\end{array}\right]-\left[\begin{array}{c}
\phi_{0} \\
\varphi_{0}
\end{array}\right]\right\|_{X^{1}} & \leqslant C\left(\|g\|_{Y^{1 / 2}}+\|h\|_{Y}\right)\left\|a_{\epsilon}-a_{0}\right\|_{L^{\infty}(\Omega)}^{1 / 2} \\
& =C\left\|\left[\begin{array}{l}
g \\
h
\end{array}\right]\right\|_{X}\left\|a_{\epsilon}-a_{0}\right\|_{L^{\infty}(\Omega)}^{1 / 2},
\end{aligned}
$$


where $C>0$ is independent of $\epsilon$.

The next result ensures convergence of the spectrum of operators $-A_{\epsilon}$.

Proposition 3.6 [Carvalho and Piskarev 2006; Kato 1976]. The following statements hold:

(i) If $\mu_{0} \in \sigma\left(-A_{0}\right)$, there exists a sequence $\epsilon_{n} \rightarrow 0^{+}$and $\left\{\mu_{n}\right\}$, with $\mu_{n} \in \sigma\left(-A_{\epsilon_{n}}\right)$, $n \in \mathbb{N}$ such that $\mu_{n} \rightarrow \mu_{0}$ as $n \rightarrow \infty$.

(ii) If for some sequences $\epsilon_{n} \rightarrow 0^{+}$and $\mu_{n} \rightarrow \mu_{0}$ as $n \rightarrow \infty$, with $\mu_{n} \in \sigma\left(-A_{\epsilon_{n}}\right)$, $n \in \mathbb{N}$, then $\mu_{0} \in \sigma\left(-A_{0}\right)$.

Now let us establish the result which treats convergence for resolvent operators. Proposition 3.7. For each $\phi \in(\pi / 2, \pi)$, there exists a constant $C=C(\phi)>0$ such that

$$
\sup _{\lambda \in \Sigma_{\phi}}\left\|\left(\lambda I+A_{\epsilon}\right)^{-1}-\left(\lambda I+A_{0}\right)^{-1}\right\|_{\mathscr{L}\left(X, X^{1}\right)} \leqslant C\left\|a_{\epsilon}-a_{0}\right\|_{L^{\infty}(\Omega)}^{1 / 2} .
$$

Proof. We can see that

$$
\begin{aligned}
\left(\lambda I+A_{\epsilon}\right)^{-1}-\left(\lambda I-A_{0}\right)^{-1} & =\left(\lambda I+A_{\epsilon}\right)^{-1} A_{\epsilon}\left[A_{0}^{-1}-A_{\epsilon}^{-1}\right] A_{0}\left(\lambda I+A_{0}\right)^{-1} \\
& =A_{\epsilon}\left(\lambda I+A_{\epsilon}\right)^{-1}\left[A_{0}^{-1}-A_{\epsilon}^{-1}\right] A_{0}\left(\lambda I+A_{0}\right)^{-1} .
\end{aligned}
$$

Notice that, for $\lambda \in \Sigma_{\phi} \subset \rho\left(-A_{\epsilon}\right)$, we have

$$
A_{\epsilon}\left(\lambda I+A_{\epsilon}\right)^{-1}=\left[\left(\lambda I+A_{\epsilon}\right) A_{\epsilon}^{-1}\right]^{-1}=\left[\lambda A_{\epsilon}^{-1}-I\right]^{-1},
$$

and, therefore,

$$
\left\|A_{\epsilon}\left(\lambda I+A_{\epsilon}\right)^{-1}\right\|_{\mathscr{L}\left(X^{1}\right)} \leqslant C, \lambda \in \Sigma_{\phi},
$$

for some $C>0$ independent of $\epsilon$.

By (3-25)-(3-27), we have the existence of a constant $C>0$ (independent of $\epsilon$ and of $\lambda \in \Sigma_{\phi}$ ) such that

$$
\left\|\left(\lambda I+A_{\epsilon}\right)^{-1}-\left(\lambda I+A_{0}\right)^{-1}\right\|_{\mathscr{L}\left(X, X^{1}\right)} \leqslant C\left\|a_{\epsilon}-a_{0}\right\|_{L^{\infty}(\Omega)}^{1 / 2} .
$$

To finish this section, we will make a spectral analysis, where we will give a characterization, as well as a rate of convergence, as $\epsilon \rightarrow 0$, for the eigenvalues associated with the operators $A_{\epsilon}$.

Let $\gamma$ be a closed, rectifiable, simple and oriented counterclockwise curve in $\rho\left(-A_{0}\right)$ around $\mu_{0} \in \sigma\left(A_{0}\right)$ which has index 1 relative to $\gamma$. From part (ii) of Proposition 3.6, it is easy to see that there is an $\epsilon_{\gamma}>0$ such that the trace of $\gamma$ is in $\rho\left(A_{\epsilon}\right)$, for $\epsilon \in\left[0, \epsilon_{\gamma}\right]$. We define the spectral projection in $X$

$$
Q_{\epsilon}\left(\mu_{0}\right)=\frac{1}{2 \pi i} \int_{\gamma}\left(\lambda I+A_{\epsilon}\right)^{-1} d \lambda,
$$


and, for $\mu \in \mathbb{C}$ such that $(1 /(2 \pi i)) \int_{\gamma}(\lambda-\mu)^{-1} d \lambda=1$, we define the generalized eigenspace associated with $\mu, W\left(\mu,-A_{\epsilon}\right)=Q_{\epsilon}\left(\mu_{0}\right)(X), \epsilon \in\left[0, \epsilon_{\gamma}\right]$. Furthermore, $Q_{\epsilon}\left(\mu_{0}\right)$ is compact and $\operatorname{dim} W\left(\mu,-A_{\epsilon}\right)=\operatorname{rank}\left(Q_{\epsilon}\left(\mu_{0}\right)\right)<\infty$.

Related to the rate of convergence, the following result holds.

Proposition 3.8. The family of operators $Q_{\epsilon}\left(\mu_{0}\right): X \rightarrow X$ converges uniformly to $Q_{0}\left(\mu_{0}\right): X \rightarrow X$, as $\epsilon \rightarrow 0^{+}$. Moreover,

$$
\left\|Q_{\epsilon}\left(\mu_{0}\right)-Q_{0}\left(\mu_{0}\right)\right\| \mathscr{L}_{(X)} \leqslant C\left\|a_{\epsilon}-a_{0}\right\|_{L^{\infty}(\Omega)}^{1 / 2},
$$

and

$$
\left\|A_{\epsilon} Q_{\epsilon}\left(\mu_{0}\right)-A_{0} Q_{0}\left(\mu_{0}\right)\right\|_{\mathscr{L}(X)} \leqslant C\left\|a_{\epsilon}-a_{0}\right\|_{L^{\infty}(\Omega)}^{1 / 2},
$$

where $C>0$ independent of $\epsilon$.

Proof. Since

$$
Q_{\epsilon}\left(\mu_{0}\right)-Q_{0}\left(\mu_{0}\right)=\frac{1}{2 \pi i} \int_{\gamma}\left[\left(\lambda I+A_{\epsilon}\right)^{-1}-\left(\lambda I+A_{0}\right)^{-1}\right] d \lambda,
$$

we can use Proposition 3.7 to guarantee the estimate (3-28).

To prove (3-29), it is sufficient to use (3-26) and (3-28).

Remark 3.9. If $\mu_{0}$ is an isolated eigenvalue for $A_{0}$, we may define $Q_{\epsilon}\left(\mu_{0}\right)$ as above and it follows from Proposition 3.6 that there exists $\mu_{\epsilon}$, which is an eigenvalue of $A_{\epsilon}$ such that $\mu_{\epsilon} \rightarrow \mu_{0}$, as $\epsilon \rightarrow 0^{+}$. Hence $Q_{\epsilon}\left(\mu_{0}\right)=Q_{\epsilon}\left(\mu_{\epsilon}\right)$. We still have from Proposition 3.8 that

$$
\left\|Q_{\epsilon}\left(\mu_{\epsilon}\right) Q_{0}\left(\mu_{0}\right)-Q_{0}\left(\mu_{0}\right)\right\|_{\mathscr{L}(X)} \leqslant C\left\|a_{\epsilon}-a_{0}\right\|_{L^{\infty}(\Omega)}^{1 / 2}
$$

and that $Q_{\epsilon}\left(\mu_{\epsilon}\right) Q_{0}\left(\mu_{0}\right)$ is an isomorphism between $R\left(Q_{0}\left(\mu_{0}\right)\right)$ and $R\left(Q_{\epsilon}\left(\mu_{\epsilon}\right)\right)$.

The next result deals with the characterization and rate of convergence of the eigenvalues associated to operators $A_{\epsilon}$.

Theorem 3.10. For each $\epsilon \in[0,1]$, the eigenvalues of the operator $A_{\epsilon}$ are given by

$$
\lambda_{\epsilon, n}^{ \pm}=\frac{\mu_{\epsilon, n}^{\delta}}{2} \pm i \frac{\sqrt{4 \mu_{\epsilon, n}-\mu_{\epsilon, n}^{2 \delta}}}{2}, \quad n \in \mathbb{N},
$$

where $\mu_{\epsilon, n}, n \in \mathbb{N}$, denotes the eigenvalues of the operator $\Lambda_{\epsilon}$. Furthermore, if $\operatorname{Ker}\left(\lambda_{0, n}^{ \pm} I-A_{0}\right)=R\left(Q_{0}\left(\lambda_{0, n}^{ \pm}\right)\right)$, then

$$
\left|\lambda_{\epsilon, n}^{ \pm}-\lambda_{0, n}^{ \pm}\right| \leqslant C_{n}\left\|a_{\epsilon}-a_{0}\right\|_{L^{\infty}(\Omega)}^{1 / 2}, \quad n \in \mathbb{N},
$$

for some constant $C_{n}>0$, independent of $\epsilon$. 
Proof. To study the spectral problem for the operator $A_{\epsilon}$, we consider the equation

$$
A_{\epsilon}\left[\begin{array}{l}
\phi \\
\varphi
\end{array}\right]=\lambda\left[\begin{array}{l}
\phi \\
\varphi
\end{array}\right]
$$

that is,

$$
\lambda^{2} \phi-\lambda \Lambda_{\epsilon}^{\delta} \phi+\Lambda_{\epsilon} \phi=0,
$$

whose solutions are the eigenvectors $\left\{\phi_{\epsilon, n}\right\}$ of $\Lambda_{\epsilon}$ :

$$
\lambda^{2} \phi_{\epsilon, n}-\mu_{\epsilon, n}^{\delta} \lambda \phi_{\epsilon, n}+\mu_{\epsilon, n} \phi_{\epsilon, n}=0 .
$$

In this way, the corresponding eigenvalues $\left\{\lambda_{\epsilon, n}^{ \pm}\right\}$of $A_{\epsilon}$ are the solutions of the equation

$$
\lambda^{2}-\mu_{\epsilon, n}^{\delta} \lambda+\mu_{\epsilon, n}=0
$$

and they are given by (3-30).

Moreover, by the above remark and Proposition 3.8, we have that, for each $\epsilon>0$, there exists $\left[\begin{array}{ll}\phi & \varphi\end{array}\right]^{T} \in R\left(Q_{0}\right),\left\|\left[\begin{array}{ll}\phi & \varphi\end{array}\right]^{T}\right\|_{X^{1}}=1$, such that $Q_{\epsilon}\left[\begin{array}{ll}\phi & \varphi\end{array}\right]^{T}$ is an eigenvector of $A_{\epsilon}$ associated to $\lambda_{\epsilon}$ and

$$
\left|\lambda_{\epsilon, n}^{ \pm}-\lambda_{0, n}^{ \pm}\right|
$$

and the proof is completed.

\section{Rate of convergence of resolvents of linearized operators}

In this section we will study the rate of convergence of the resolvents of operators which corresponds to linearizations of (1-7) around equilibria.

It is known that the Nemytskiı map $f^{e}(u):=f(u), u \in Y^{1 / 2}$, is Fréchet continuously differentiable. Moreover, if $\left\{u_{\epsilon}\right\}$ converges to $u_{0}$ in $Y^{1 / 2}$ and $0 \notin$ $\sigma\left(\Lambda_{0}-\left(f^{e}\right)^{\prime}\left(u_{0}\right)\right)$, then $\left(\left(f^{e}\right)^{\prime}\left(u_{\epsilon}\right)\right) \Lambda_{\epsilon}^{-1}$ converges to $\left(\left(f^{e}\right)^{\prime}\left(u_{0}\right)\right) \Lambda_{0}^{-1}$ in the uniform operator topology of $\mathscr{L}(Y)$; see, for instance, [Arrieta et al. 2013]. Hence the Nemytskiŭ map $F$ is Fréchet continuously differentiable. Moreover, if $u_{\epsilon} \rightarrow u_{0}$ in $X$ and $0 \notin \sigma\left(A_{0}-F^{\prime}\left(u_{0}\right)\right)$, then

$$
\left(F^{\prime}\left(u_{\epsilon}\right)\right) A_{\epsilon}^{-1} \rightarrow\left(F^{\prime}\left(u_{0}\right)\right) A_{0}^{-1} \text { in } \mathscr{L}(X) .
$$

Lemma 4.1. We assume $u_{\epsilon} \rightarrow u_{0}$ in $X$ and $0 \notin \sigma\left(A_{0}-F^{\prime}\left(u_{0}\right)\right)$. Then there exists $\epsilon_{0}>0$ such that the net of operators

$$
\left\{A_{\epsilon}^{1} / 2\left(A_{\epsilon}-F^{\prime}\left(u_{\epsilon}\right)\right)^{-1}: \epsilon \in[0,1]\right\}
$$


is uniformly bounded in $\mathscr{L}(X)$ and

$$
\left\|A_{\epsilon}^{1} / 2\left(A_{\epsilon}-F^{\prime}\left(u_{\epsilon}\right)\right)^{-1}-A_{0}^{1} / 2\left(A_{0}-F^{\prime}\left(u_{0}\right)\right)^{-1}\right\|_{\mathscr{L}(X)} \leqslant C\left\|a_{\epsilon}-a_{0}\right\|_{L^{\infty}(\Omega)}^{1 / 2},
$$

where $C>0$ is independent of $\epsilon$.

Proof. The proof follows from the identity

$$
A_{\epsilon}^{1 / 2}\left(A_{\epsilon}-F^{\prime}\left(u_{\epsilon}\right)\right)^{-1}=A_{\epsilon}^{-1 / 2}\left(I-F^{\prime}\left(u_{\epsilon}\right) A_{\epsilon}^{-1}\right)^{-1}
$$

and by (4-1).

\section{Rate of convergence of the linear and nonlinear semigroups}

Since the operators $A_{\epsilon}, \epsilon \in[0,1]$, are self-adjoint and $A_{\epsilon}^{-1}$ converges uniformly to $A_{0}^{-1}$ as $\epsilon \rightarrow 0^{+}$, for each $\alpha<\lambda_{1}^{0}\left(\lambda_{1}^{0}\right.$ the first eigenvalue of $A_{0}$ ), there exists $C>0$, independent of $\epsilon \in[0,1]$, such that

$$
\left\|e^{-A_{\epsilon} t}\right\|_{\mathscr{L}(X)} \leqslant C e^{-\alpha t} t^{-1 / 2}, \quad t>0, \epsilon \in[0,1] .
$$

Theorem 5.1. If $\theta \in\left(0, \frac{1}{2}\right]$ and $\alpha<\lambda_{1}^{0}$, there exists $C>0$, independent of $\epsilon$, such that

$$
\left\|e^{-A_{\epsilon} t}-e^{-A_{0} t}\right\| \mathscr{L}_{(X)} \leqslant C e^{-\alpha t}\left\|a_{\epsilon}-a_{0}\right\|_{L^{\infty}(\Omega)}^{2 \theta} t^{-(1 / 2+\theta)}
$$

for all $t>0$ and $\epsilon \in[0,1]$.

Proof. Considering the linear semigroup

$$
e^{-A_{\epsilon} t}=\frac{1}{2 \pi i} \int_{\Gamma} e^{\lambda t}\left(\lambda I+A_{\epsilon}\right)^{-1} d \lambda, \quad \epsilon \in[0,1],
$$

where $\Gamma$ is the boundary of sector $\Sigma_{-\omega, \phi}=\{\lambda \in \mathbb{C}:|\arg (\lambda+\omega)| \leqslant \phi\}$ with $\pi / 2<\phi<\pi$, oriented in such a way that the imaginary part of $\lambda$ increases as $\lambda$ runs in $\Gamma$.

The estimate

$$
\left\|e^{-A_{\epsilon} t}-e^{-A_{0} t}\right\|_{\mathscr{L}(X)} \leqslant\left\|e^{-A_{\epsilon} t}\right\|_{\mathscr{L}(X)}+\left\|e^{-A_{0} t}\right\|_{\mathscr{L}(X)} \leqslant C e^{-\alpha t} t^{-1 / 2}
$$

follows by (5-1).

On the other hand, using Proposition 3.7, we have

$$
\left\|e^{-A_{\epsilon} t}-e^{-A_{0} t}\right\|_{\mathscr{L}(X)} \leqslant C e^{-\alpha t}\left\|a_{\epsilon}-a_{0}\right\|_{L^{\infty}(\Omega)}^{1 / 2} t^{-1} .
$$

Therefore, for $\theta \in\left(0, \frac{1}{2}\right]$, we obtain

$$
\begin{aligned}
\left\|e^{-A_{\epsilon} t}-e^{-A_{0} t}\right\|_{\mathscr{L}(X)} & \leqslant C e^{-\alpha(1-2 \theta) t} t^{-1 / 2(1-2 \theta)} e^{-\alpha(2 \theta) t}\left\|a_{\epsilon}-a_{0}\right\|_{L^{\infty}(\Omega)}^{2 \theta} t^{-2 \theta} \\
& \leqslant C e^{-\alpha t}\left\|a_{\epsilon}-a_{0}\right\|_{L^{\infty}(\Omega)}^{2 \theta} t^{-(1 / 2+\theta)} .
\end{aligned}
$$

where $C>0$ is independent of $\epsilon$. 
Theorem 5.2. Let $\left[\begin{array}{ll}u_{\epsilon} & v_{\epsilon}\end{array}\right]^{T},\left[\begin{array}{ll}u & v\end{array}\right]^{T} \in X$, and $\theta \in\left(0, \frac{1}{2}\right)$. Then there are positive constants $C$ and $L_{f}$ such that

$$
\left\|T_{\epsilon}(t)\left[\begin{array}{c}
u_{\epsilon} \\
v_{\epsilon}
\end{array}\right]-T_{0}(t)\left[\begin{array}{c}
u \\
v
\end{array}\right]\right\|_{X} \leqslant C e^{L t} t^{-(1 / 2+\theta)}\left(\left\|\left[\begin{array}{c}
u_{\epsilon} \\
v_{\epsilon}
\end{array}\right]-\left[\begin{array}{c}
u \\
v
\end{array}\right]\right\|_{X}+\left\|a_{\epsilon}-a_{0}\right\|_{L^{\infty}(\Omega)}^{\theta}\right)
$$

for all $t \geqslant 0$.

Proof. For $t \geqslant 0$ and $\left[\begin{array}{ll}u_{\epsilon} & v_{\epsilon}\end{array}\right]^{T} \in X$ we have

$$
T_{\epsilon}(t)\left[\begin{array}{c}
u_{\epsilon} \\
v_{\epsilon}
\end{array}\right]=e^{-A_{\epsilon} t}\left[\begin{array}{c}
u_{\epsilon} \\
v_{\epsilon}
\end{array}\right]+\int_{0}^{t} e^{-A_{\epsilon}(t-s)} f\left(T_{\epsilon}(s)\left[\begin{array}{c}
u_{\epsilon} \\
v_{\epsilon}
\end{array}\right]\right) d s, \quad \epsilon \in[0,1]
$$

and therefore

$$
\begin{aligned}
&\left\|T_{\epsilon}(t)\left[\begin{array}{c}
u_{\epsilon} \\
v_{\epsilon}
\end{array}\right]-T_{0}(t)\left[\begin{array}{c}
u \\
v
\end{array}\right]\right\|_{X} \\
& \leqslant\left\|e^{-A_{\epsilon} t}\left[\begin{array}{c}
u_{\epsilon} \\
v_{\epsilon}
\end{array}\right]-e^{-A_{0} t}\left[\begin{array}{c}
u \\
v
\end{array}\right]\right\|_{X}+\int_{0}^{t} \| e^{-A_{\epsilon}(t-s)} f\left(T_{\epsilon}(s)\left[\begin{array}{c}
u_{\epsilon} \\
v_{\epsilon}
\end{array}\right]\right) \\
&-e^{-A_{0}(t-s)} f\left(T_{0}(s)\left[\begin{array}{c}
u \\
v
\end{array}\right]\right) \|_{X} d s .
\end{aligned}
$$

From (5-1) and (5-2) we get

$$
\begin{aligned}
\| e^{-A_{\epsilon} t}\left[\begin{array}{c}
u_{\epsilon} \\
v_{\epsilon}
\end{array}\right] & -e^{-A_{0} t}\left[\begin{array}{l}
u \\
v
\end{array}\right] \|_{X} \\
& \leqslant C t^{-(1 / 2+\theta)}\left\|\left[\begin{array}{c}
u_{\epsilon} \\
v_{\epsilon}
\end{array}\right]-\left[\begin{array}{c}
u \\
v
\end{array}\right]\right\|_{X}+C\left\|a_{\epsilon}-a_{0}\right\|_{L^{\infty}(\Omega)}^{2 \theta} t^{-(1 / 2+\theta)} .
\end{aligned}
$$

We still have

$$
\begin{gathered}
\int_{0}^{t}\left\|e^{-A_{\epsilon}(t-s)} f\left(T_{\epsilon}(s)\left[\begin{array}{c}
u_{\epsilon} \\
v_{\epsilon}
\end{array}\right]\right)-e^{-A_{0}(t-s)} f\left(T_{0}(s)\left[\begin{array}{c}
u \\
v
\end{array}\right]\right)\right\|_{X} d s \\
\leqslant C L_{f} \int_{0}^{t}(t-s)^{-1 / 2} e^{-\alpha(t-s)}\left\|T_{\epsilon}(s)\left[\begin{array}{c}
u_{\epsilon} \\
v_{\epsilon}
\end{array}\right]-T_{0}(s)\left[\begin{array}{c}
u \\
v
\end{array}\right]\right\|_{X} d s \\
+C\left\|a_{\epsilon}-a_{0}\right\|_{L^{\infty}(\Omega)}^{2 \theta} \int_{0}^{t}(t-s)^{-(1 / 2+\theta)} e^{-\alpha(t-s)} d s .
\end{gathered}
$$


Substituting (5-6) and (5-7) in (5-5), it follows that

$$
\begin{aligned}
\left\|T_{\epsilon}(t)\left[\begin{array}{c}
u_{\epsilon} \\
v_{\epsilon}
\end{array}\right]-T_{0}(t)\left[\begin{array}{c}
u \\
v
\end{array}\right]\right\|_{X} \\
\leqslant C\left(\left\|\left[\begin{array}{c}
u_{\epsilon} \\
v_{\epsilon}
\end{array}\right]-\left[\begin{array}{c}
u \\
v
\end{array}\right]\right\|_{X}+\left\|a_{\epsilon}-a_{0}\right\|_{L^{\infty}(\Omega)}^{2 \theta}\right) t^{-(1 / 2+\theta)} e^{-\alpha t} \\
+C L_{f} \int_{0}^{t}(t-s)^{-1 / 2} e^{-\alpha(t-s)}\left\|T_{\epsilon}(s)\left[\begin{array}{c}
u_{\epsilon} \\
v_{\epsilon}
\end{array}\right]-T_{0}(s)\left[\begin{array}{c}
u \\
v
\end{array}\right]\right\|_{X} d s .
\end{aligned}
$$

Thus the singular Gronwall inequality (see Lemma 7.1.1 in [Henry 1981]) guarantees the existence of a constant $L>0$ such that

$$
\begin{aligned}
\left\|T_{\epsilon}(t)\left[\begin{array}{c}
u_{\epsilon} \\
v_{\epsilon}
\end{array}\right]-T_{0}(t)\left[\begin{array}{c}
u \\
v
\end{array}\right]\right\|_{X} \\
\quad \leqslant C e^{L_{f} t} t^{-(1 / 2+\theta)}\left(\left\|\left[\begin{array}{c}
u_{\epsilon} \\
v_{\epsilon}
\end{array}\right]-\left[\begin{array}{c}
u \\
v
\end{array}\right]\right\|_{X}+\left\|a_{\epsilon}-a_{0}\right\|_{L^{\infty}(\Omega)}^{\theta}\right) .
\end{aligned}
$$

\section{Rate of convergence of the equilibria and of the linearizations}

Now we will work to control the behavior of equilibria in terms of $\left\|a_{\epsilon}-a_{0}\right\|_{L^{\infty}(\Omega)}^{1 / 2}$. First, we will give the definition of equilibrium of problem (1-7).

Definition 6.1. The equilibrium solutions of (1-7) are the functions that solve the stationary

$$
A_{\epsilon} w^{\epsilon}=F\left(w^{\epsilon}\right), \quad \epsilon \in[0,1] .
$$

For each $\epsilon \in[0,1]$, we denote by $\mathscr{E}_{\epsilon}$ the set of the equilibrium solutions of (1-7). We say that an equilibrium $w_{*}^{\epsilon}$ of (1-7) is hyperbolic if the spectrum $\sigma\left(A_{\epsilon}-F^{\prime}\left(w_{*}^{\epsilon}\right)\right)$ of $A_{\epsilon}-F^{\prime}\left(w_{*}^{\epsilon}\right)$ is disjoint from the imaginary axis.

We start by proving the upper semicontinuity of the family of equilibria.

Proposition 6.2. The family $\left\{\mathscr{E}_{\epsilon}: \epsilon \in[0,1]\right\}$ is upper semicontinuous at $\epsilon=0$.

Proof. Since $\mathscr{E}_{\epsilon}$ is contained in $\mathscr{A}_{\epsilon}, \sup \left\{\left\|w^{\epsilon}\right\|_{X}: w^{\epsilon} \in \mathscr{E}_{\epsilon}, \epsilon \in[0,1]\right\}<\infty$. Using the fact that $F: X \rightarrow Y \times Y$ is bounded, for each $w^{\epsilon} \in \mathscr{E}_{\epsilon}$, we have that $w^{\epsilon}=A_{\epsilon}^{-1} F\left(w^{\epsilon}\right)$, and the result follows from the uniform convergence of $A_{\epsilon}^{-1}$ to $A_{0}^{-1}$.

The proof of lower semicontinuity requires additional assumptions. We need to assume that the equilibrium points of (1-7) are stable under perturbation. This stability under perturbation will be given by the hyperbolicity.

Proposition 6.3. Any hyperbolic point of $\mathscr{E}_{0}$ is isolated. 
Proof. We note that $w_{*} \in \mathscr{E}_{0}$ is a solution of (6-1) if and only if $w_{*}$ is a fixed point of

$$
\Psi(w):=\left(A_{0}-F^{\prime}\left(w_{*}\right)\right)^{-1}\left(F(w)-F^{\prime}\left(w_{*}\right) w\right) .
$$

If we show that, for some $r>0, \Psi: \bar{B}_{r}\left(w_{*}\right) \rightarrow \bar{B}_{r}\left(w_{*}\right)$ is a contraction, where $\bar{B}_{r}\left(w_{*}\right):=\left\{w \in X:\left\|w-w_{*}\right\|_{X} \leqslant r\right\}$, then $w_{*}$ is a unique element in $\bar{B}_{r}\left(w_{*}\right) \cap \mathscr{E}_{0}$ and, consequently, is isolated. In fact, letting $r>0$ and $u, v \in \bar{B}_{r}\left(u_{*}\right)$, we observe by (4-1) that

$$
\begin{aligned}
\|\Psi(u)-\Psi(v)\|_{X} & \leqslant\left\|\left(A_{0}-F^{\prime}\left(w_{*}\right)\right)^{-1}\right\| \mathscr{L}(X)\left\|\left(F(u)-F(v)-F^{\prime}\left(w_{*}\right)(u-v)\right)\right\|_{X} \\
& \leqslant C r\|u-v\|_{X} .
\end{aligned}
$$

Thus, choosing $r$ such that $C r<1$, we have $\Psi$ is a contraction. We can see that, if $v \in \bar{B}_{r}\left(w_{*}\right)$, then $\left\|\Psi(v)-w_{*}\right\|_{X}=\left\|\Psi(v)-\Psi\left(w_{*}\right)\right\|_{X} \leqslant C\left\|v-w_{*}\right\|_{X}<r$, for some constant $C \in[0,1)$. Then $\Psi\left(\bar{B}_{r}\left(w_{*}\right)\right) \subset \bar{B}_{r}\left(w_{*}\right)$. This implies that $\Psi$ has a unique fixed point in $\bar{B}_{r}\left(w_{*}\right)$ and the proof is complete.

Corollary 6.4. The set $\mathscr{E}_{0}$ has at most a finite number of hyperbolic points.

Proof. It follows directly of the compactness of $\mathscr{E}_{\epsilon}$.

Now we are going to study the convergence properties of resolvent operators of the form $\left(A_{\epsilon}+V_{\epsilon}\right)^{-1}$, with $V_{\epsilon} \in \mathscr{L}\left(X^{1}, X\right)$. This is because we are interested in comparing the resolvent operators of the linearization around equilibrium.

The convergence of resolvents of $A_{\epsilon}+V_{0}$ follows from the convergence of resolvents of $A_{\epsilon}$ (see Proposition 3.5) and the lemma below, whose proof is immediate.

Lemma 6.5. The operator $A_{\epsilon}+V_{0}, \epsilon \in[0,1]$, satisfies the identity

$$
\begin{aligned}
\left(A_{\epsilon}+V_{0}\right)^{-1}- & \left(A_{0}+V_{0}\right)^{-1} \\
& =\left[I-\left(A_{\epsilon}+V_{0}\right)^{-1} V_{0}\right]\left(A_{\epsilon}^{-1}-A_{0}^{-1}\right)\left[I-V_{0}\left(A_{0}+V_{0}\right)^{-1}\right] .
\end{aligned}
$$

Theorem 6.6. Let us consider $w_{*}$ a hyperbolic of $\mathscr{E}_{0}$ with $0 \notin \sigma\left(A_{0}-f^{\prime}\left(w_{*}\right)\right)$. Then there exist $\epsilon_{1}>0$ and $r>0$ such that problem (1-7) has exactly one equilibrium solution $w_{*}^{\epsilon}$ in $\bar{B}_{r}\left(w_{*}\right):=\left\{w \in X:\left\|w-w_{*}\right\|_{X} \leqslant r\right\}$ for $\epsilon \in\left[0, \epsilon_{1}\right]$. Furthermore, $\left\|w_{*}^{\epsilon}-w_{*}\right\|_{X} \leqslant C\left\|a_{\epsilon}-a_{0}\right\|_{L^{\infty}(\Omega)}^{1 / 2}$ for some $C>0$ independent of $\epsilon$.

Proof. The hyperbolicity of $w_{*}$ means that $\sigma\left(A_{\epsilon}-f^{\prime}\left(w_{*}\right)\right)$ is disjoint from the imaginary axis. Thus, by Lemma 4.1 , we can guarantee the existence of a constant $C>0$ such that

$$
\left\|\left(A_{\epsilon}-F^{\prime}\left(w_{*}\right)\right)^{-1}\right\|_{\mathscr{L}(X)} \leqslant C, \quad \epsilon \in[0,1] .
$$

We have that $w^{\epsilon}$ is a solution of (6-1) if and only if it is a fixed point of the map

$$
\Psi_{\epsilon}(\omega):=\left(A_{\epsilon}-F^{\prime}\left(w_{*}\right)\right)^{-1}\left(F(w)-F^{\prime}\left(w_{*}\right) w\right) .
$$


From Lemma 4.1, we get that $A_{\epsilon}^{1 / 2}\left(A_{\epsilon}-F^{\prime}\left(w_{*}\right)\right)^{-1}$ converges uniformly to $A_{0}^{1 / 2}\left(A_{0}-F^{\prime}\left(w_{*}\right)\right)^{-1}$, which implies

$$
\Psi_{\epsilon}\left(w_{*}\right) \rightarrow \Psi_{0}\left(w_{*}\right) \quad \text { in } X .
$$

Now we will prove the existence of $r>0$ and $\epsilon_{1} \in[0,1]$ such that $\Psi_{\epsilon}$ is a contraction of $\bar{B}_{r}\left(w_{*}\right)=\left\{w \in X:\left\|w-w_{*}\right\|_{X}<r\right\}$ into itself, uniformly in $\left[0, \epsilon_{1}\right]$. In fact, first we will see that $\Psi_{\epsilon}$ is a contraction map. For this, we take $u^{\epsilon}$ and $v^{\epsilon}$ in $\bar{B}_{r}\left(w_{*}\right)$. In this way,

$$
\begin{aligned}
& \left\|\Psi_{\epsilon}\left(u^{\epsilon}\right)-\Psi_{\epsilon}\left(v^{\epsilon}\right)\right\|_{X} \\
& \quad=\left\|\left(A_{\epsilon}-F^{\prime}\left(w_{*}\right)\right)^{-1}\left[F\left(u^{\epsilon}\right)-F\left(v^{\epsilon}\right)-F^{\prime}\left(w_{*}\right)\left(u^{\epsilon}-v^{\epsilon}\right)\right]\right\|_{X} \\
& \quad \leqslant\left\|\left(A_{\epsilon}-F^{\prime}\left(w_{*}\right)\right)^{-1}\right\|_{\mathscr{L}\left(X, X^{1}\right)}\left\|F\left(u^{\epsilon}\right)-F\left(v^{\epsilon}\right)-F^{\prime}\left(u_{*}\right)\left(u^{\epsilon}-v^{\epsilon}\right)\right\|_{X} \\
& \quad=\left\|A_{\epsilon}^{-1}\left(I-F^{\prime}\left(w_{*}\right)^{-1} A_{\epsilon}^{-1}\right)\right\|_{\mathscr{L}\left(X, X^{1}\right)}\left\|F\left(u^{\epsilon}\right)-F\left(v^{\epsilon}\right)-F^{\prime}\left(u_{*}\right)\left(u^{\epsilon}-v^{\epsilon}\right)\right\|_{X},
\end{aligned}
$$

and, according to Proposition 3.5 and (4-1), there exist $C>0$ and $\epsilon_{1}>0$ such that (6-3) $\left\|\Psi_{\epsilon}\left(u^{\epsilon}\right)-\Psi_{\epsilon}\left(v^{\epsilon}\right)\right\|_{X^{1}} \leqslant C \delta\left\|u^{\epsilon}-v^{\epsilon}\right\|_{X}, \quad$ for all $\delta>0$ and all $\epsilon \in\left[0, \epsilon_{1}\right]$.

Therefore, choosing $\delta$ such that $C \delta \leqslant a<1$, it follows that $\Psi_{\epsilon}$ is a contraction as claimed.

Let us show now that $\Psi_{\epsilon}\left(\bar{B}_{r}\left(w_{*}\right)\right) \subset \bar{B}_{r}\left(w_{*}\right)$. Taking $u^{\epsilon} \in \bar{B}_{r}\left(w_{*}\right)$, we obtain by (6-3) that

$$
\begin{aligned}
\left\|\Psi_{\epsilon}\left(u^{\epsilon}\right)-w_{*}\right\|_{X} & \leqslant\left\|\Psi_{\epsilon}\left(u^{\epsilon}\right)-\Psi_{\epsilon}\left(w_{*}\right)\right\|_{X}+\left\|\Psi_{\epsilon}\left(w_{*}\right)-w_{*}\right\|_{X} \\
& \leqslant a\left\|u^{\epsilon}-w_{*}\right\|_{X}+\left\|\Psi_{\epsilon}\left(w_{*}\right)-w_{*}\right\|_{X} \\
& \leqslant a r+\left\|\Psi_{\epsilon}\left(w_{*}\right)-w_{*}\right\|_{X}, \quad \text { for all } \epsilon \in(0, \bar{\epsilon}] .
\end{aligned}
$$

It follows from Lemma 4.1 that there exists $\epsilon_{1}>0$ such that

$$
\left\|\Psi_{\epsilon}\left(w_{*}\right)-w_{*}\right\|_{X} \leqslant r / 2, \quad \text { for all } \epsilon \in\left[0, \epsilon_{1}\right] .
$$

Combining (6-4) and (6-5), and considering $a \leqslant 1 / 2$, we deduce that

$$
\left\|\Psi_{\epsilon}\left(u^{\epsilon}\right)-w_{*}\right\|_{X} \leqslant r, \quad \text { for all } \epsilon \in\left[0, \epsilon_{1}\right],
$$

and, therefore, $\Psi_{\epsilon}: \bar{B}_{r}\left(w_{*}\right) \rightarrow \bar{B}_{r}\left(w_{*}\right)$ is a contraction, for all $\epsilon \in\left[0, \epsilon_{1}\right]$. Hence, there exists a fixed point of $\Psi_{\epsilon}$ in $\bar{B}_{r}\left(w_{*}\right)$, which we will call $w_{*}^{\epsilon}$.

Finally, we will find an estimate of the difference $w_{*}^{\epsilon}-w_{*}$ in terms of $\| a_{\epsilon}-$ $a_{0} \|_{L^{\infty}(\Omega)}^{1 / 2}$.

Observe that $w_{*}^{\epsilon}=\Psi_{\epsilon}\left(w_{*}^{\epsilon}\right)$ and $w_{*}=\Psi_{0}\left(w_{*}\right)$. If we denote $F^{\prime}\left(w_{*}\right)=V_{0}$, we have

$$
\begin{aligned}
\left\|w_{*}^{\epsilon}-w_{*}\right\|_{X} \leqslant \|\left(\left(A_{\epsilon}\right.\right. & \left.\left.+V_{0}\right)^{-1}-\left(A_{0}+V_{0}\right)^{-1}\right)\left[F\left(w_{*}^{\epsilon}\right)+V_{0} w_{*}^{\epsilon}\right] \\
& +\left(A_{0}+V_{0}\right)^{-1}\left[F\left(w_{*}^{\epsilon}\right)-F\left(w_{*}\right)+V_{0}\left(w_{*}^{\epsilon}-w_{*}\right)\right] \|_{X} .
\end{aligned}
$$


Identity (6-2) and Proposition 3.5 give us

$$
\left\|\left(A_{\epsilon}+V_{0}\right)^{-1}-\left(A_{0}+V_{0}\right)^{-1}\right\|_{\mathscr{L}(X)} \leqslant C\left\|A_{\epsilon}^{-1}-A_{0}^{-1}\right\|_{\mathscr{L}(X)} \leqslant C\left\|a_{\epsilon}-a_{0}\right\|_{L^{\infty}(\Omega)}^{1 / 2},
$$

where the constant $C>0$ is independent of $\epsilon$.

On the other hand, denoting $z_{*}^{\epsilon}=F\left(w_{*}^{\epsilon}\right)-F\left(w_{*}\right)+V_{0}\left(w_{*}^{\epsilon}-w_{*}\right)$ and using the differentiability of the map $F: X \rightarrow Y \times Y$ (see (4-1)), we get that, for every $r>0$, $\left\|z_{\epsilon}\right\|_{X} \leqslant r\left\|w_{*}^{\epsilon}-w_{*}\right\|_{X}$. Hence,

$$
\left\|\left(A_{0}+V_{0}\right)^{-1} z_{*}^{\epsilon}\right\|_{X} \leqslant r\left\|\left(A_{0}+V_{0}\right)^{-1}\right\| \mathscr{L}(X)\left\|w_{*}^{\epsilon}-w_{*}\right\|_{X} .
$$

Substituting (6-7) and (6-8) in (6-5) and choosing $r>0$ such that $r \|\left(A_{0}+\right.$ $\left.V_{0}\right)^{-1} \|_{\mathscr{L}(X)} \leqslant 1 / 2$, we obtain

$$
\left\|w_{*}^{\epsilon}-w_{*}\right\|_{X} \leqslant C\left\|F\left(w_{*}^{\epsilon}\right)+V_{0} w_{*}^{\epsilon}\right\|_{X}\left\|a_{\epsilon}-a_{0}\right\|_{L^{\infty}(\Omega)}^{1 / 2}+\frac{1}{2}\left\|w_{*}^{\epsilon}-w_{*}\right\|_{X},
$$

which, combined with the fact that $f$ and its derivative are limited, allows us to conclude

$$
\left\|w_{*}^{\epsilon}-w_{*}\right\|_{X} \leqslant C\left\|a_{\epsilon}-a_{0}\right\|_{L^{\infty}(\Omega)}^{1 / 2} .
$$

Remark 6.7. Notice that, by assuming that elements of $\mathscr{E}_{0}=\left\{w_{*}^{1,0}, \ldots, w_{*}^{\mathfrak{n}, 0}\right\}$ are hyperbolic, we have that the points of $\mathscr{E}_{\epsilon}=\left\{w_{*}^{1, \epsilon}, \ldots, w_{*}^{\mathfrak{n}, \epsilon}\right\}$, with $\epsilon \in(0,1]$, satisfy the estimate $\left\|w_{*}^{i, \epsilon}-w_{*}^{i, 0}\right\|_{X} \leqslant C\left\|a_{\epsilon}-a_{0}\right\|_{L^{\infty}(\Omega)}^{1 / 2}$. We still have by (4-1) that, writing $V_{\epsilon}=F^{\prime}\left(w_{*}^{\epsilon}\right)$ with $w_{*}^{\epsilon} \in \mathscr{E}_{\epsilon}, V_{\epsilon}$ converges to $V_{0}$ in the uniform topology.

Lemma 6.8. There exists a constant $C>0$, independent of $\epsilon$, such that

$$
\left\|V_{\epsilon} A_{\epsilon}^{-1}-V_{0} A_{0}^{-1}\right\|_{\mathscr{L}(X)} \leqslant C\left\|a_{\epsilon}-a_{0}\right\|_{L^{\infty}(\Omega)}^{1 / 2} .
$$

Proof. The estimate follows by the decomposition

$$
V_{\epsilon} A_{\epsilon}^{-1}-V_{0} A_{0}^{-1}=V_{\epsilon}\left(A_{\epsilon}^{-1}-A_{0}^{-1}\right)+\left(V_{\epsilon}-V_{0}\right) A_{0}^{-1},
$$

(3-25), and Theorem 6.6.

The next result shows an analogous property found in Proposition 3.5 with $A_{\epsilon}+V_{\epsilon}$ instead of $A_{\epsilon}$. This will be important in the analysis in the next section.

Proposition 6.9. Let us consider $\bar{A}_{\epsilon}=A_{\epsilon}+V_{\epsilon}$ for all $\epsilon \in[0,1]$. If $0 \in \rho\left(\bar{A}_{0}\right)$, then $0 \in \rho\left(\bar{A}_{\epsilon}\right)$, for all $\epsilon \in(0,1]$, and the following identity holds:

(6-9) $\bar{A}_{\epsilon}^{-1}-\bar{A}_{0}^{-1}$

$=\left(A_{\epsilon}^{-1}-A_{0}^{-1}\right)\left(I+V_{0} A_{0}^{-1}\right)^{-1}-A_{\epsilon}^{-1}\left(I+V_{0} A_{0}^{-1}\right)^{-1}\left(V_{\epsilon} A_{\epsilon}^{-1}-V_{0} A_{0}^{-1}\right)\left(I+V_{\epsilon} A_{\epsilon}^{-1}\right)^{-1}$.

Furthermore

$$
\left\|\bar{A}_{\epsilon}^{-1}-\bar{A}_{0}^{-1}\right\|_{\mathscr{L}(X)} \leqslant C\left\|a_{\epsilon}-a_{0}\right\|_{L^{\infty}(\Omega)}^{1 / 2},
$$

for some $C>0$ independent of $\epsilon$. 
Proof. The first part follows from Proposition 2.1. The identity (6-9) is immediate, and (6-10) follows using (6-9), Proposition 3.5, and Lemma 6.8.

The last proposition enables us to prove similar results as Proposition 3.6, Proposition 3.7, Theorem 5.1 and Theorem 5.2 for $A_{\epsilon}+V_{\epsilon}$ instead of $A_{\epsilon}$.

\section{Rate of convergence and attraction of local unstable manifolds}

The main aim of this section is the proof of the existence unstable local manifolds as a graph of a Lipschitz function, its convergence, and exponential attraction.

For each $\epsilon \in\left[0, \epsilon_{1}\right]$, let us consider $w_{*}^{\epsilon}$ to be an equilibrium solution for (1-7). We assume the existence of a constant $C>0$ such that $\left\|w_{*}^{\epsilon}-w_{*}\right\|_{X} \leqslant C\left\|a_{\epsilon}-a_{0}\right\|_{L^{\infty}(\Omega)}^{1 / 2}$, for all $\epsilon \in\left[0, \epsilon_{1}\right]$, and that $w_{*}:=w_{*}^{0}$ is hyperbolic. To deal with a neighborhood of the equilibrium point $w_{*}^{\epsilon}$, we rewrite the problems (1-7) as

$$
z_{t}^{\epsilon}+\bar{A}_{\epsilon} z^{\epsilon}=F\left(u^{\epsilon}+w_{*}^{\epsilon}\right)-F\left(w_{*}^{\epsilon}\right)-F^{\prime}\left(w_{*}^{\epsilon}\right) u^{\epsilon},
$$

where $z^{\epsilon}=u^{\epsilon}-w_{*}^{\epsilon}$ and $\bar{A}_{\epsilon}=A_{\epsilon}-F^{\prime}\left(w_{*}^{\epsilon}\right)$. With this, one can look for Proposition 3.7 with $\bar{A}_{\epsilon}$ instead of $A_{\epsilon}$.

Let $\gamma$ be a smooth, closed, simple, rectifiable curve in $\{z \in \mathbb{C}: \operatorname{Re} z>0\}$, oriented counterclockwise and such that the bounded connected component of $\mathbb{C} \backslash\{\gamma\}$ (here $\{\gamma\}$ denotes the trace of $\gamma)$ contains $\left\{z \in \sigma\left(-\bar{A}_{0}\right): \operatorname{Rez}>0\right\}$. From part (ii) of Proposition 3.6, there exists $\epsilon_{1}>0$ such that $\{\gamma\} \subset \rho\left(-\bar{A}_{\epsilon}\right)$ for all $\epsilon \in\left[0, \epsilon_{1}\right]$. We define $\bar{Q}_{\epsilon}$ by

$$
\bar{Q}_{\epsilon}=\frac{1}{2 \pi i} \int_{\gamma}\left(\lambda I-\bar{A}_{\epsilon}\right)^{-1} d \lambda, \quad \text { for all } \epsilon \in\left[0, \epsilon_{1}\right] .
$$

The operator $\bar{A}_{\epsilon}$ is self-adjoint and there exist $\beta>0$ and $C \geqslant 1$ such that

$$
\left\|e^{-\bar{A}_{\epsilon} t} \bar{Q}_{\epsilon}\right\|_{\mathscr{L}(X)} \leqslant C e^{-\beta t}, \quad \text { for all } t \geqslant 0 \text { and all } \epsilon \in\left[0, \epsilon_{1}\right]
$$

and

$$
\left\|e^{-\bar{A}_{\epsilon} t}\left(I-\bar{Q}_{\epsilon}\right)\right\|_{\mathscr{L}(X)} \leqslant C t^{-1 / 2} e^{-\beta t}, \quad t>0 .
$$

Using the decomposition $X=\bar{Q}_{\epsilon} X \oplus\left(I-\bar{Q}_{\epsilon}\right) X$ (the solution $z^{\epsilon}$ of (7-1) can be decomposed as $\left.z^{\epsilon}=\bar{Q}_{\epsilon} z^{\epsilon}+\left(I-\bar{Q}_{\epsilon}\right) z^{\epsilon}\right)$, we rewrite (7-1) as

$$
\left\{\begin{array}{l}
\frac{d}{d t}\left(\bar{Q}_{\epsilon} z^{\epsilon}\right)+\bar{A}_{\epsilon} \bar{Q}_{\epsilon} z^{\epsilon}=H_{\epsilon}\left(\bar{Q}_{\epsilon} z^{\epsilon},\left(I-\bar{Q}_{\epsilon}\right) z^{\epsilon}\right), \\
\frac{d}{d t}\left[\left(I-\bar{Q}_{\epsilon}\right) z^{\epsilon}\right]+\bar{A}_{\epsilon}\left(I-\bar{Q}_{\epsilon}\right) z^{\epsilon}=G_{\epsilon}\left(\bar{Q}_{\epsilon} z^{\epsilon},\left(I-\bar{Q}_{\epsilon}\right) z^{\epsilon}\right),
\end{array}\right.
$$

where

$$
\begin{aligned}
& H_{\epsilon}\left(\bar{Q}_{\epsilon} z^{\epsilon},\left(I-\bar{Q}_{\epsilon}\right) z^{\epsilon}\right) \\
& :=\bar{Q}_{\epsilon}\left[F\left(\bar{Q}_{\epsilon} z^{\epsilon}+\left(I-\bar{Q}_{\epsilon}\right) z^{\epsilon}+w_{*}^{\epsilon}\right)-F\left(w_{*}^{\epsilon}\right)-F^{\prime}\left(w_{*}^{\epsilon}\right)\left(\bar{Q}_{\epsilon} z^{\epsilon}+\left(I-\bar{Q}_{\epsilon}\right) z^{\epsilon}\right)\right],
\end{aligned}
$$


and

$$
G_{\epsilon}\left(\bar{Q}_{\epsilon} z^{\epsilon},\left(I-\bar{Q}_{\epsilon}\right) z^{\epsilon}\right)
$$

$:=\left(I-\bar{Q}_{\epsilon}\right)\left[F\left(\bar{Q}_{\epsilon} z^{\epsilon}+\left(I-\bar{Q}_{\epsilon}\right) z^{\epsilon}+w_{*}^{\epsilon}\right)-F\left(w_{*}^{\epsilon}\right)-F^{\prime}\left(w_{*}^{\epsilon}\right)\left(\bar{Q}_{\epsilon} z^{\epsilon}+\left(I-\bar{Q}_{\epsilon}\right) z^{\epsilon}\right)\right]$.

The functions $H_{\epsilon}$ and $G_{\epsilon}$ are continuously differentiable with

$$
H_{\epsilon}(0,0)=G_{\epsilon}(0,0)=0
$$

and $H_{\epsilon}^{\prime}(0,0)=0=G_{\epsilon}^{\prime}(0,0) \in \mathscr{L}(X)$. For simplicity of notation, we write $\omega^{\epsilon}=\bar{Q}_{\epsilon} z^{\epsilon}$ and $\vartheta^{\epsilon}=\left(I-\bar{Q}_{\epsilon}\right) z^{\epsilon}$. Hence, given $\rho>0$, there exist $\epsilon_{1}>0$ and $r>0$ such that if $\left\|\omega^{\epsilon}\right\|_{\bar{Q}_{\epsilon} X}+\left\|\vartheta^{\epsilon}\right\|_{\left(I-\bar{Q}_{\epsilon}\right) X}<r$ and $\epsilon \in\left[0, \epsilon_{1}\right]$, then

$$
\left\|H_{\epsilon}\left(\omega^{\epsilon}, \vartheta^{\epsilon}\right)\right\|_{\bar{Q}_{\epsilon} X} \leqslant \rho \quad \text { and } \quad\left\|G_{\epsilon}\left(\omega^{\epsilon}, \vartheta^{\epsilon}\right)\right\|_{\left(I-\bar{Q}_{\epsilon}\right) X} \leqslant \rho,
$$

$$
\left\|H_{\epsilon}\left(\omega^{\epsilon}, \vartheta^{\epsilon}\right)-H_{\epsilon}\left(\bar{\omega}^{\epsilon}, \bar{\vartheta}^{\epsilon}\right)\right\|_{\bar{Q}_{\epsilon} X} \leqslant \rho\left(\left\|\omega^{\epsilon}-\bar{\omega}^{\epsilon}\right\|_{\bar{Q}_{\epsilon} X}+\left\|\vartheta^{\epsilon}-\bar{\vartheta}^{\epsilon}\right\|_{\left(I-\bar{Q}_{\epsilon}\right) X}\right)
$$

and

$$
\left\|G_{\epsilon}\left(\omega^{\epsilon}, \vartheta^{\epsilon}\right)-G_{\epsilon}\left(\bar{\omega}^{\epsilon}, \bar{\vartheta}^{\epsilon}\right)\right\|_{\left(I-\bar{Q}_{\epsilon}\right) X} \leqslant \rho\left(\left\|\omega^{\epsilon}-\bar{\omega}^{\epsilon}\right\|_{\bar{Q}_{\epsilon} X}+\left\|\vartheta^{\epsilon}-\bar{\vartheta}^{\epsilon}\right\|_{\left(I-\bar{Q}_{\epsilon}\right) X}\right) .
$$

Considering the coupled system (7-2), we can show an unstable manifold theorem using similar arguments to those in the results of Chapter 6 in [Henry 1981].

Theorem 7.1. There exists a map $s_{*}^{\epsilon}: \bar{Q}_{\epsilon} X \rightarrow\left(I-\bar{Q}_{\epsilon}\right) X$ such that the unstable manifold of $w_{*}^{\epsilon}$ is given by

$$
W^{u}\left(w_{*}^{\epsilon}\right)=\left\{(\omega, \vartheta) \in X: \vartheta=s_{*}^{\epsilon}(\omega), \omega \in \bar{Q}_{\epsilon} X\right\} .
$$

The map $s_{*}^{\epsilon}$ satisfies

$$
\left\|s_{*}^{\epsilon}\right\|:=\sup _{\omega \in \bar{Q}_{\epsilon} X}\left\|s_{*}^{\epsilon}(\omega)\right\|_{X} \leqslant C, \quad\left\|s_{*}^{\epsilon}(\omega)-s_{*}^{\epsilon}(\tilde{\omega})\right\|_{X} \leqslant \bar{C}\|\omega-\tilde{\omega}\|_{\bar{Q}_{\epsilon} X},
$$

where $C>0$ is a constant independent of $\epsilon$, and for $\theta \in\left(0, \frac{1}{2}\right)$ there exists a $C>0$, independent of $\epsilon$, such that

$$
\left\|s_{*}^{\epsilon}-s_{*}^{0}\right\| \mid \leqslant C\left\|a_{\epsilon}-a_{0}\right\|_{L^{\infty}(\Omega)}^{2 \theta} .
$$

Furthermore, there exists $\rho_{1}>0, C>0$ (independent of $\epsilon$ ), and $t_{0}>0$ such that, for any solution $\left(\omega^{\epsilon}(t), \vartheta^{\epsilon}(t)\right) \in X, t \in\left[t_{0}, \infty\right)$, of (7-2), we have

(7-9) $\left\|\vartheta^{\epsilon}(t)-s_{*}^{\epsilon}\left(\omega^{\epsilon}(t)\right)\right\|_{X} \leqslant C e^{-\rho_{1}\left(t-t_{0}\right)}\left\|\vartheta^{\epsilon}\left(t_{0}\right)-s_{*}^{\epsilon}\left(\omega^{\epsilon}\left(t_{0}\right)\right)\right\|_{X}, \quad$ for all $t \geqslant t_{0}$.

Proof. We consider the set

$$
\Sigma_{\epsilon}=\left\{s: \bar{Q}_{\epsilon} X^{1} \rightarrow\left(I-\bar{Q}_{\epsilon}\right) X:\|s\| \leqslant C,\|s(\omega)-s(\tilde{\omega})\|_{X} \leqslant C\|\omega-\tilde{\omega}\|_{\bar{Q}_{\epsilon} X}\right\} .
$$

It is not difficult to see that $\left(\Sigma_{\epsilon},\|\cdot\|\right)$ is a complete metric space. 
Given $s \in \Sigma_{\epsilon}$ and $\eta \in \bar{Q}_{\epsilon} X$, we denote by $\omega^{\epsilon}(t)=\psi(t, \tau, \eta, s)$ the solution of

$$
\left\{\begin{array}{l}
\omega_{t}^{\epsilon}(t)+B_{\epsilon} \omega^{\epsilon}(t)=H_{\epsilon}\left(\omega^{\epsilon}(t), s\left(\omega^{\epsilon}(t)\right)\right), \quad t<\tau \\
\omega^{\epsilon}(\tau)=\eta
\end{array}\right.
$$

We define $\Psi_{\epsilon}: \Sigma_{\epsilon} \rightarrow \Sigma_{\epsilon}$ by

$$
\Psi_{\epsilon}(s) \eta=\int_{-\infty}^{\tau} e^{-\widetilde{A}_{\epsilon}(\tau-\xi)} G_{\epsilon}\left(\omega^{\epsilon}(\xi), s\left(\omega^{\epsilon}(\xi)\right)\right) d \xi .
$$

According to Theorem 7.1 in [Arrieta et al. 2013], we can deduce that $\Psi_{\epsilon}$ is a contraction. Therefore, there is a fixed point $s_{*}^{\epsilon}=\Psi\left(s_{*}^{\epsilon}\right)$ in $\Sigma_{\epsilon}$.

Now we shall prove that the graph of $s_{*}^{\epsilon}\left\{\left(\omega^{\epsilon}, s_{*}^{\epsilon}\left(\omega^{\epsilon}\right)\right): \omega^{\epsilon} \in \bar{Q}_{\epsilon} X\right\}$ is invariant for (7-2), in the sense that initial data for (7-2) in $\left\{\left(\omega^{\epsilon}, s_{*}^{\epsilon}\left(\omega^{\epsilon}\right)\right): \omega^{\epsilon} \in \bar{Q}_{\epsilon} X\right\}$ lead to solutions in this space. In fact, we take $\left(\omega_{0}^{\epsilon}, \vartheta_{0}^{\epsilon}\right) \in W^{u}\left(w_{*}^{\epsilon}\right)\left(\vartheta_{0}^{\epsilon}=s_{*}^{\epsilon}\left(\omega_{0}^{\epsilon}\right)\right)$. We denote by $\omega_{*}^{\epsilon}(t)$ the solution of the initial value problems

$$
\left\{\begin{array}{l}
\frac{d}{d t}\left(\bar{Q}_{\epsilon} z^{\epsilon}\right)+\bar{A}_{\epsilon} \bar{Q}_{\epsilon} z^{\epsilon}=H_{\epsilon}\left(\omega^{\epsilon}, s_{*}^{\epsilon}\left(\omega^{\epsilon}\right)\right), \\
\omega^{\epsilon}(0)=\omega_{0}^{\epsilon},
\end{array}\right.
$$

where $z^{\epsilon}=\omega^{\epsilon}+\vartheta^{\epsilon} \in \bar{Q}_{\epsilon} X \oplus\left(I-\bar{Q}_{\epsilon}\right) X$. This defines a curve $\left(\omega_{*}^{\epsilon}(t), s_{*}^{\epsilon}\left(\omega_{*}^{\epsilon}(t)\right)\right) \in$ $W^{u}\left(w_{*}^{\epsilon}\right), t \in \mathbb{R}$. Also, the unique solution of

$$
\frac{d}{d t}\left[\left(I-\bar{Q}_{\epsilon}\right) z^{\epsilon}\right]+\bar{A}_{\epsilon}\left(I-\bar{Q}_{\epsilon}\right) z^{\epsilon}=G_{\epsilon}\left(\omega^{\epsilon}, s_{*}^{\epsilon}\left(\omega^{\epsilon}\right)\right),
$$

which remains bounded as $t \rightarrow-\infty$, is

$\vartheta_{*}^{\epsilon}(t)=\left(I-\bar{Q}_{\epsilon}\right) z_{*}^{\epsilon}(t)=\int_{-\infty}^{t} e^{\bar{A}_{\epsilon}\left(I-\bar{Q}_{\epsilon}\right)(t-\xi)} G_{\epsilon}\left(\omega_{*}^{\epsilon}(\xi), s_{*}^{\epsilon}\left(\omega_{*}^{\epsilon}(\xi)\right)\right) d \xi=s_{*}^{\epsilon}\left(\omega_{*}^{\epsilon}(t)\right)$.

Therefore $\left(\omega_{*}^{\epsilon}(t), s_{*}^{\epsilon}\left(\omega_{*}^{\epsilon}(t)\right)\right)$ is a solution of the system (7-2) through the point $\left(\omega_{0}^{\epsilon}, \vartheta_{0}^{\epsilon}\right)$, proving the invariance of the graph of $s_{*}^{\epsilon}$.

To show (7-8), we can proceed as in the proof of Proposition 6.1 in [Arrieta et al. 2009].

Finally, the proof that the graph of $s_{*}^{\epsilon}$ is the unstable manifold that attracts exponentially, uniformly in $\epsilon$, that is, the inequality (7-9) holds, follows by similar arguments to those in the proof of (A.8) in [Bruschi et al. 2006].

Now we are able to prove our main result.

\section{Proof of Theorem 1.1}

The purpose of this section is to emphasize the proof of our main result. For this, we return to Theorem 1.1 to establish its proof. 
Proof of Theorem 1.1. This proof follows by Theorem 5.2, Theorem 6.6, and Theorem 7.1 jointly with Theorems 2.1 and 2.2, and Corollary 2.1 in Chapter 8 of [Babin and Vishik 1989].

\section{Acknowledgments}

The authors thank A.N. Carvalho for constructive conversations about this problem.

\section{References}

[Arrieta et al. 2009] J. M. Arrieta, A. N. Carvalho, and G. Lozada-Cruz, "Dynamics in dumbbell domains, III: Continuity of attractors", J. Differential Equations 247:1 (2009), 225-259. MR 2010i:37191 Zbl 1172.35034

[Arrieta et al. 2013] J. M. Arrieta, F. D. M. Bezerra, and A. N. Carvalho, "Rate of convergence of global attractors of some perturbed reaction-diffusion problems", Topol. Methods Nonlinear Anal. 41:2 (2013), 229-254.

[Babin and Vishik 1989] A. V. Babin and M. I. Vishik, Аттракторы еволючионных уравнений, Nauka, Moscow, 1989. Translated and revised as Attractors of evolution equations, Studies in Mathematics and its Applications 25, North-Holland, Amsterdam, 1992. MR 92f:58101 Zbl 0804.58003

[Bruschi et al. 2006] S. M. Bruschi, A. N. Carvalho, J. W. Cholewa, and T. Dlotko, "Uniform exponential dichotomy and continuity of attractors for singularly perturbed damped wave equations", J. Dynam. Differential Equations 18:3 (2006), 767-814. MR 2008b:37132 Zbl 1103.35020

[Carvalho and Cholewa 2002a] A. N. Carvalho and J. W. Cholewa, "Attractors for strongly damped wave equations with critical nonlinearities", Pacific J. Math. 207:2 (2002), 287-310. MR 2004b: 35023 Zbl 1060.35082

[Carvalho and Cholewa 2002b] A. N. Carvalho and J. W. Cholewa, "Local well posedness for strongly damped wave equations with critical nonlinearities", Bull. Austral. Math. Soc. 66:3 (2002), 443-463. MR 2004b:35228 Zbl 1020.35059

[Carvalho and Piskarev 2006] A. N. Carvalho and S. Piskarev, "A general approximation scheme for attractors of abstract parabolic problems", Numer. Funct. Anal. Optim. 27:7-8 (2006), 785-829. MR 2007h:37122 Zbl 1110.35012

[Chen and Triggiani 1989] S. P. Chen and R. Triggiani, "Proof of extensions of two conjectures on structural damping for elastic systems", Pacific J. Math. 136:1 (1989), 15-55. MR 90g:47071 Zbl 0633.47025

[Cholewa and Dlotko 2006] J. W. Cholewa and T. Dlotko, "Strongly damped wave equation in uniform spaces”, Nonlinear Anal. 64:1 (2006), 174-187. MR 2006f:35183 Zbl 1083.35066

[Egorov and Shubin 1988] Y. V. Egorov and M. A. Shubin, “Линейные дифференциальные уравнения с частными производными, 1: Основы классической теории”, Itogi Nauki i Tekhniki. Ser. Sovrem. Probl. Mat. Fund. Napr. 30 (1988), 5-255. Translated as Partial differential equations, 1: Foundations of the classical theory, Encyclopaedia of Mathematical Sciences 30, Springer, Berlin, 1992. MR 93a:35004b Zbl 0657.35002

[Hale 1988] J. K. Hale, Asymptotic behavior of dissipative systems, Mathematical Surveys and Monographs 25, Amer. Math. Soc., Providence, RI, 1988. MR 89g:58059 Zbl 0642.58013

[Henry 1981] D. Henry, Geometric theory of semilinear parabolic equations, Lecture Notes in Mathematics 840, Springer, Berlin, 1981. MR 83j:35084 Zbl 0456.35001 
[Kato 1976] T. Kato, Perturbation theory for linear operators, 2nd ed., Grundlehren der Mathematischen Wissenschaften 132, Springer, Berlin, 1976. MR 53 \#11389 Zbl 0342.47009

[Pazy 1983] A. Pazy, Semigroups of linear operators and applications to partial differential equations, Applied Mathematical Sciences 44, Springer, New York, 1983. MR 85g:47061 Zbl 0516.47023

[Sobolevskii 1961] P. E. Sobolevskii, “Об уравнениях параболического типа в банаховом пространстве”, Trudy Moskov. Mat. Obšč. 10 (1961), 297-350. Translated as "Equations of parabolic type in a Banach space" in Amer. Math. Soc. Transl. 49 (1966), 1-62. MR 25 \#5297 Zbl 0141.32701

[Suggs 2009] J. D. Suggs, "Decay for nonlinear wave equations with variable coefficients", Undergraduate honors thesis project, University of Tennessee, Knoxville, TN, 2009, Available at http:// trace.tennessee.edu/utk_chanhonoproj/1326.

[Webb 1980] G. F. Webb, "Existence and asymptotic behavior for a strongly damped nonlinear wave equation”, Canad. J. Math. 32:3 (1980), 631-643. MR 81i:35116 Zbl 0414.35046

[Wu and Li 2011] J. Wu and S. Li, "Stabilization of wave equation with variable coefficients by nonlinear boundary feedback”, J. Syst. Sci. Complex. 24:5 (2011), 875-882. MR 2012j:35224 Zbl 1249.35184

Received October 3, 2012. Revised March 4, 2013.

FÁGNER Dias ARARUNA

DEPARTAMENTO DE MATEMÁTICA

UNIVERSIDAdE FEDERAL DA PARAÍBA

JoÃo PESSOA, PB 58051-900

BRAZIL

fagner@mat.ufpb.br

Flank DaVid Morais BEZERRA

Departamento de Matemática

UNIVERSIDADE FEDERAL DA PARAÍBA

JoÃo PESSOA, PB 58051-900

BRAZIL

flank@mat.ufpb.br 


\title{
PACIFIC JOURNAL OF MATHEMATICS
}

\author{
msp.org/pjm
}

Founded in 1951 by E. F. Beckenbach (1906-1982) and F. Wolf (1904-1989)

\section{EDITORS}

V. S. Varadarajan (Managing Editor)

Department of Mathematics

University of California

Los Angeles, CA 90095-1555

pacific@math.ucla.edu

Paul Balmer

Department of Mathematics

University of California

Los Angeles, CA 90095-1555

balmer@math.ucla.edu

Daryl Cooper

Department of Mathematics

University of California

Santa Barbara, CA 93106-3080 cooper@math.ucsb.edu

Jiang-Hua Lu

Department of Mathematics

The University of Hong Kong

Pokfulam Rd., Hong Kong jhlu@maths.hku.hk
Don Blasius

Department of Mathematics University of California

Los Angeles, CA 90095-1555

blasius@math.ucla.edu

Robert Finn

Department of Mathematics Stanford University

Stanford, CA 94305-2125

finn@math.stanford.edu

Sorin Popa

Department of Mathematics

University of California

Los Angeles, CA 90095-1555 popa@math.ucla.edu

Paul Yang

Department of Mathematics Princeton University

Princeton NJ 08544-1000

yang@math.princeton.edu

\section{PRODUCTION}

Silvio Levy, Scientific Editor, production@msp.org

\section{SUPPORTING INSTITUTIONS}

ACADEMIA SINICA, TAIPEI

CALIFORNIA INST. OF TECHNOLOGY

INST. DE MATEMÁTICA PURA E APLICADA

KEIO UNIVERSITY

MATH. SCIENCES RESEARCH INSTITUTE

NEW MEXICO STATE UNIV.

OREGON STATE UNIV.

\author{
STANFORD UNIVERSITY \\ UNIV. OF BRITISH COLUMBIA \\ UNIV. OF CALIFORNIA, BERKELEY \\ UNIV. OF CALIFORNIA, DAVIS \\ UNIV. OF CALIFORNIA, LOS ANGELES \\ UNIV. OF CALIFORNIA, RIVERSIDE \\ UNIV. OF CALIFORNIA, SAN DIEGO \\ UNIV. OF CALIF., SANTA BARBARA
}

\author{
Vyjayanthi Chari \\ Department of Mathematics \\ University of California \\ Riverside, CA 92521-0135 \\ chari@math.ucr.edu \\ Kefeng Liu \\ Department of Mathematics \\ University of California \\ Los Angeles, CA 90095-1555 \\ liu@math.ucla.edu \\ Jie Qing \\ Department of Mathematics \\ University of California \\ Santa Cruz, CA 95064 \\ qing@cats.ucsc.edu
}

These supporting institutions contribute to the cost of publication of this Journal, but they are not owners or publishers and have no responsibility for its contents or policies.

See inside back cover or msp.org/pjm for submission instructions.

The subscription price for 2013 is US \$400/year for the electronic version, and \$485/year for print and electronic.

Subscriptions, requests for back issues and changes of subscribers address should be sent to Pacific Journal of Mathematics, P.O. Box 4163, Berkeley, CA 94704-0163, U.S.A. The Pacific Journal of Mathematics is indexed by Mathematical Reviews, Zentralblatt MATH, PASCAL CNRS Index, Referativnyi Zhurnal, Current Mathematical Publications and Web of Knowledge (Science Citation Index).

The Pacific Journal of Mathematics (ISSN 0030-8730) at the University of California, c/o Department of Mathematics, 798 Evans Hall \#3840, Berkeley, CA 94720-3840, is published twelve times a year. Periodical rate postage paid at Berkeley, CA 94704, and additional mailing offices. POSTMASTER: send address changes to Pacific Journal of Mathematics, P.O. Box 4163, Berkeley, CA 94704-0163.

PJM peer review and production are managed by EditFLOW ${ }^{\circledR}$ from Mathematical Sciences Publishers.

\section{PUBLISHED BY}

mathematical sciences publishers

nonprofit scientific publishing

http://msp.org/

(C) 2013 Mathematical Sciences Publishers 


\section{PACIFIC JOURNAL OF MATHEMATICS}

Volume $266 \quad$ No. $2 \quad$ December 2013

Rate of attraction for a semilinear wave equation with variable coefficients and

critical nonlinearities

FÁGNER DiAs ARARUNA and FLANK DAVID MORAIS BEZERRA

The Brin-Thompson groups $s V$ are of type $\mathrm{F}_{\infty}$

Martin G. Fluch, Marco Marschler, Stefan Witzel and

MATTHEW C. B. ZAREMSKY

Ideal decompositions of a ternary ring of operators with predual

MASAYOSHI KANEDA

A study of real hypersurfaces with Ricci operators in 2-dimensional complex space 305 forms

Dong Ho Lim, WoOn Ha SoHn and Hyunjung Song

On commensurability of fibrations on a hyperbolic 3-manifold

HideTOSHI MASAI

Multiplicative Dirac structures

CRISTIÁN ORTIZ

On the finite generation of a family of Ext modules

TONY J. PuthenPuRAKAL

Index formulae for Stark units and their solutions

XAVIER-FranÇOIS ROBLOT

The short time asymptotics of Nash entropy

GUOYI XU

Several splitting criteria for vector bundles and reflexive sheaves

STEPHEN S.-T. YAU and FEI YE

The minimal volume orientable hyperbolic 3-manifold with 4 cusps

KEN'ICHI YOSHIDA

On the Witten rigidity theorem for string ${ }^{c}$ manifolds

JIANQING YU and BO LIU 